\title{
Perturbation Theory and Optimality Conditions for the Best Multilinear Rank Approximation of a Tensor
}

Lars Eldén and Berkant Savas

\section{Linköping University Post Print}

N.B.: When citing this work, cite the original article.

Original Publication:

Lars Eldén and Berkant Savas, Perturbation Theory and Optimality Conditions for the Best Multilinear Rank Approximation of a Tensor, 2011, SIAM Journal on Matrix Analysis and Applications, (32), 4, 1422-1450.

http://dx.doi.org/10.1137/110823298

Copyright: Society for Industrial and Applied Mathematics http://www.siam.org/

Postprint available at: Linköping University Electronic Press

http://urn.kb.se/resolve?urn=urn:nbn:se:liu:diva-72910 


\title{
PERTURBATION THEORY AND OPTIMALITY CONDITIONS FOR THE BEST MULTILINEAR RANK APPROXIMATION OF A TENSOR $^{*}$
}

\author{
LARS ELDÉN ${ }^{\dagger}$ AND BERKANT SAVAS $\ddagger$
}

\begin{abstract}
The problem of computing the best rank- $(p, q, r)$ approximation of a third order tensor is considered. First the problem is reformulated as a maximization problem on a product of three Grassmann manifolds. Then expressions for the gradient and the Hessian are derived in a local coordinate system at a stationary point, and conditions for a local maximum are given. A first order perturbation analysis is performed using the Grassmann manifold framework. The analysis is illustrated in a few examples, and it is shown that the perturbation theory for the singular value decomposition is a special case of the tensor theory.
\end{abstract}

Key words. tensor, multilinear rank, best rank- $(p, q, r)$ approximation, perturbation theory, first order optimality conditions, second order optimality conditions, Grassmann manifold, stationary point

AMS subject classifications. 65F99, 65K10, 15A69, 14M15, 47A55

DOI. $10.1137 / 110823298$

1. Introduction. The problem of approximating a given tensor $\mathcal{A} \in \mathbb{R}^{l \times m \times n}$ by another tensor $\mathcal{B}$ of equal dimensions but of lower rank,

$$
\min _{\mathcal{B}}\|\mathcal{A}-\mathcal{B}\|
$$

occurs in various applications, e.g., signal processing $[2,3,7]$, analytical chemistry [27], bioinformatics [23], computer vision [31], machine learning [21, 19], neuroscience [22], quantum chemistry [17], and pattern classification [25]. See also the bibliography of the recent survey [18]. The multilinear approximation of a given tensor is also the basis of the Tucker model $[29,30]$. There is no unique definition of the rank of a tensor, as opposed to the case of matrices. Here we will deal with the concept of multilinear rank defined by Hitchcock [14]. The multilinear rank of a tensor is also considered in $[6,8]$. We assume that $\operatorname{rank}(\mathcal{B})=(p, q, r)$, which means that the tensor $\mathcal{B}$ can be written as a product of a core tensor $\mathcal{F}$ and three matrices,

$$
\mathcal{B}=(X, Y, Z) \cdot \mathcal{F}, \quad \mathcal{B}(i, j, k)=\sum_{\lambda=1}^{p} \sum_{\mu=1}^{q} \sum_{\nu=1}^{r} x_{i \lambda} y_{j \mu} z_{k \nu} f_{\lambda \mu \nu}
$$

where $X \in \mathbb{R}^{l \times p}, Y \in \mathbb{R}^{m \times q}$, and $Z \in \mathbb{R}^{n \times r}$ have full column rank and the tensor $\mathcal{F}$ has dimensions $p \times q \times r$. It is not restrictive to assume that $X, Y$, and $Z$ have orthonormal columns, as any nonorthonormality may be incorporated into $\mathcal{F}$. Then,

*Received by the editors February 3, 2011; accepted for publication (in revised form) by B. Hendrickson September 6, 2011; published electronically December 8, 2011.

http://www.siam.org/journals/simax/32-4/82329.html

$\dagger$ Department of Mathematics, Linköping University, SE-581 83 Linköping, Sweden (laeld@math. liu.se).

$\ddagger$ Institute for Computational Engineering and Sciences, The University of Texas at Austin, Austin, TX 78712 (berkant.savas@liu.se). This author's work was supported by the Swedish Research Council, Dnr 2008-7145, and the Institute for Computational Engineering and Sciences at The University of Texas at Austin. 
the best multilinear rank- $(p, q, r)$ approximation problem can be written as

$$
\min _{\mathcal{F}, X, Y, Z}\|\mathcal{A}-(X, Y, Z) \cdot \mathcal{F}\| \quad \text { subject to } \quad X^{\top} X=I, \quad Y^{\top} Y=I, \quad Z^{\top} Z=I .
$$

The tensor approximation problem in terms of the multilinear product (1.1) is illustrated in Figure 1.1. The corresponding low rank approximation problem for a given matrix $A$ has an explicit solution in terms of the singular value decomposition (SVD) of the matrix $A$. It can be shown that the minimization problem (1.2) in the tensor case is well defined [6], [8, Corollary 4.5], but there is no known closed form solution. However, several iterative approaches have been proposed (see $[12,26,6,16]$ ); in $[12,26]$ we developed Newton and quasi-Newton methods for solving the minimization problem when stated as an optimization problem on Grassmann manifolds.

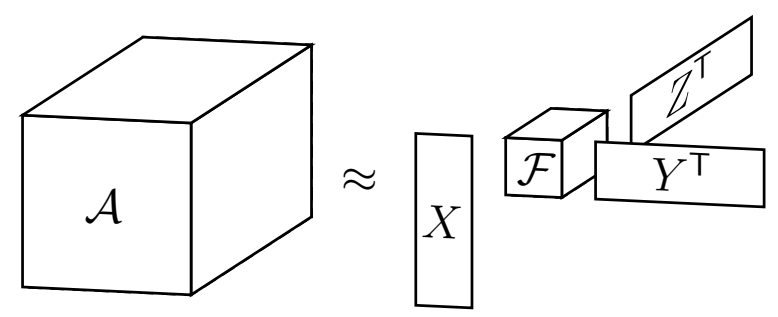

FIG. 1.1. The approximation of a tensor $\mathcal{A}$ by another tensor $(X, Y, Z) \cdot \mathcal{F}$ of lower multilinear rank.

To contribute to the understanding of the properties of (1.2) we derive and analyze first and second order conditions for a local minimum of the optimization problem, and develop a perturbation theory, which is of vital importance for the development and design of algorithms for (1.2). In particular we generalize the concept of "gap," which governs the sensitivity of the singular subspaces in the matrix case [28], to corresponding quantities for the tensor case. The analysis is based on the differential-geometric framework developed in [12]. The orthonormality constraints on the unknown matrices $X, Y$, and $Z$ are taken into account by formulating the problem as a maximization problem on a product of three Grassmann manifolds. Within this framework it is also straightforward to generalize the derivations to higher (than three) order tensors. We also compare the properties of the solution of (1.2) to those of the matrix low rank approximation problem and point out similarities and differences. In particular, we demonstrate that the perturbation theory for the SVD is a special case of our analysis.

For simplicity of presentation, we will describe the theory mainly in terms of third order tensors or shortly 3 -tensors. In view of the lack of a standard terminology and notation in the field of tensor computations we define the concepts used in section 2 . In section 3 we formulate the best rank- $(p, q, r)$ approximation problem and related expressions for the gradient and the Hessian on a product of three Grassmann manifolds. We formulate the first and second order optimality conditions for a local maximum point in section 4 , and in section 5 the perturbation theory is developed. Throughout we give examples that illustrate the application of the theory to special cases. In section 6 we present the conclusions of the paper.

2. Tensor concepts and identities. For simplicity of notation and presentation, we will mostly present the various concepts using examples in terms of 3-tensors. Some more general definitions are given in $[5,1,8]$. We will use roman letters written 
with a calligraphic font to denote tensors, e.g., $\mathcal{A}$ and $\mathcal{B}$. Capital roman letters will denote matrices (2-tensors), e.g., $X, Y$, and $Z$. Bold face capital roman letters will denote linear operators, e.g., $\mathbf{H}$ and $\mathbf{L}$. We will mostly use lower case roman letters to denote tensor dimensions, e.g., $l, m, n ; i, j, \mu$, and $\nu$ will denote tensor subscripts and summation indexes. In a few places we will also use lower case roman letters to denote vectors.

In the approximation problem (1.2) the tensor may be viewed as a multidimensional array of numbers with associated mathematical operations. Let $\mathcal{A}$ denote a tensor in $\mathbb{R}^{l \times m \times n}$. The three "dimensions" of the tensor are referred to as modes. Usually we will not consider a given tensor as a multilinear operator, and therefore there is no need to make a distinction between contravariant and covariant tensor modes in the tensor notation. A particular tensor element will be denoted in both "MATLAB-like" notation and with standard subscripts, i.e., $\mathcal{A}(i, j, k)=a_{i j k}$. A subtensor obtained by fixing one of the indices is called a slice, i.e., $\mathcal{A}(i,:,:)$ is referred to as a mode-1 slice. A fibre is a subtensor, where all indices but one are fixed, e.g. $\mathcal{A}(i,:, k)$ is called a mode-2 fibre.

It is customary in numerical linear algebra to write out column vectors with the elements arranged vertically, and row vectors with the elements horizontally. This becomes inconvenient when we are dealing with more than two modes. Therefore we will not make a notational distinction between mode- 1 , mode- 2 , and mode- 3 vectors, and we will allow ourselves to write all vectors organized vertically. It will be clear from the context to which mode the vectors belong. However, when we are dealing with matrices, we will often talk of them as consisting of column vectors. When in the following we use tensors, matrices, and vectors in operations that we will define, it is assumed that the dimensions of the respective quantities are conforming so that all the operations are well defined.

2.1. Multilinear tensor-matrix multiplication. The multilinear multiplication of a tensor $\mathcal{A} \in \mathbb{R}^{l \times m \times n}$ with matrices $U \in \mathbb{R}^{p \times l}, V \in \mathbb{R}^{q \times m}$, and $W \in \mathbb{R}^{r \times n}$ is written $^{1}$

$$
\mathcal{B}=(U, V, W) \cdot \mathcal{A}
$$

where the entries of $\mathcal{B}$, which is a $p \times q \times r$ tensor, are given by

$$
\mathcal{B}(i, j, k)=b_{i j k}=\sum_{\lambda=1}^{l} \sum_{\mu=1}^{m} \sum_{\nu=1}^{n} u_{i \lambda} v_{j \mu} w_{k \nu} a_{\lambda \mu \nu} .
$$

This operation may also be considered as a multilinear transformation of the $l \times m \times n$ tensor $\mathcal{A}$ to the $p \times q \times r$ tensor $\mathcal{B}$. This operation is multilinear since the transformation is linear in each of $U, V$, or $W$ separately.

When a tensor is multiplied by a single matrix in mode $i$, say, we will call the operation the mode-i multilinear multiplication (or mode- $i$ product) of a tensor by a matrix. For concreteness let $i=1$. Then we will write the mode- 1 product of a tensor $\mathcal{A} \in \mathbb{R}^{l \times m \times n}$ by a matrix $U \in \mathbb{R}^{p \times l}$ as

$$
\mathbb{R}^{q \times m \times n} \ni \mathcal{B}=(U)_{1} \cdot \mathcal{A}, \quad \mathcal{B}(i, j, k)=\sum_{\lambda=1}^{l} u_{i \lambda} a_{\lambda j k} .
$$

\footnotetext{
${ }^{1}$ This is the notation introduced in [8]. An alternative notation was given in [5].
} 
This means that all mode- 1 fibers in the 3 -tensor $\mathcal{A}$ are multiplied by the matrix $U$. More generally, mode- $i$ multiplication by a matrix $X$ means that all mode- $i$ fibers are multiplied by the matrix $X$.

One can show that for integers $i \neq j$, mode- $i$ and mode- $j$ multiplications commute, i.e., $(U)_{i} \cdot\left((V)_{j} \cdot \mathcal{A}\right)=(V)_{j} \cdot\left((U)_{i} \cdot \mathcal{A}\right)$, so we can write

$$
(U, V)_{i, j} \cdot \mathcal{A}=(U)_{i} \cdot\left((V)_{j} \cdot \mathcal{A}\right) .
$$

One can also show that

$$
\left(U_{1}\right)_{i} \cdot\left(\left(U_{2}\right)_{i} \cdot \mathcal{A}\right)=\left(U_{1} U_{2}\right)_{i} \cdot \mathcal{A},
$$

where the product $U_{1} U_{2}$ is standard matrix multiplication. For convenience, when there are transposes involved in the multiplication, we use the following notation:

$$
\left(X^{\top}\right)_{i} \cdot \mathcal{A}=\mathcal{A} \cdot(X)_{i} .
$$

One can also write the standard matrix multiplication of three matrices in the form

$$
X F Y^{\top}=(X, Y) \cdot F
$$

where $F$ may be simultaneously considered as a matrix and a 2 -tensor.

2.2. Inner product, tensor product, and contracted product. In the following we will define a few more fundamental tensor operations that are used throughout the paper. Given two tensors $\mathcal{A}$ and $\mathcal{B}$ of same dimensions, we define the inner product as

$$
\langle\mathcal{A}, \mathcal{B}\rangle=\sum_{i, j, k} a_{i j k} b_{i j k}
$$

The corresponding tensor norm or Frobenius norm is given by

$$
\|\mathcal{A}\|=\langle\mathcal{A}, \mathcal{A}\rangle^{1 / 2} .
$$

As in the matrix case the Frobenius norm of a tensor is invariant under orthogonal transformations, i.e., $\|\mathcal{A}\|=\|(U, V, W) \cdot \mathcal{A}\|$, for orthogonal matrices $U, V$, and $W$. This follows immediately from the fact that mode- $i$ multiplication by an orthogonal matrix does not change the Euclidean length of the mode- $i$ fibers.

The tensor product or outer product of two tensors, $\mathcal{A} \in \mathbb{R}^{l \times m \times n}$ and $\mathcal{B} \in \mathbb{R}^{p \times q \times r}$, say, is a tensor of higher dimensionality, here a 6 -tensor,

$$
\mathbb{R}^{l \times m \times n \times p \times q \times r} \ni \mathcal{C}=\mathcal{A} \otimes \mathcal{B}, \quad c_{i j k \lambda \mu \nu}=a_{i j k} b_{\lambda \mu \nu} .
$$

In the rest of the paper we will reserve the symbol $\otimes$ to mean the Kronecker product of matrices, which can be considered as the tensor product of two matrices (2-tensors), followed by a particular matricization of the 4 -tensor. In this paper it is sufficient to define the mode- $i$ matricization of the tensor $\mathcal{A} \in \mathbb{R}^{l \times m \times n}$ as the matrix $A^{(i)}$ consisting of all the mode- $i$ fibers. For example, with $i=2$ we have

$$
A^{(2)}=[\mathcal{A}(1,:, 1) \mathcal{A}(1,:, 2) \cdots \mathcal{A}(l,:, n)] \in \mathbb{R}^{m \times l n} .
$$

There are different mode- 2 matricizations with respect to the particular ordering of the mode-2 fibers $\mathcal{A}(i,:, k)$. The ordering is unimportant from a theoretical point 
of view but from a practical point of view some orderings have benefits over others, in particular when matricization is performed on tensor-matrix products $[12$, section $2.2]$.

The inner product (2.6) can be considered as a special case of a contracted product of two tensors. If $\mathcal{A}$ and $\mathcal{B}$ are 3 -tensors of conforming dimensions, we define, using essentially the notation of [1],

$$
\begin{array}{rlrl}
\mathcal{C} & =\langle\mathcal{A}, \mathcal{B}\rangle_{1}, \\
D & =\langle\mathcal{A}, \mathcal{B}\rangle_{1,2}, \\
c_{j k \mu \nu} & =\sum_{\lambda} a_{\lambda j k} b_{\lambda \mu \nu} \\
d_{k \nu} & =\sum_{\lambda, \mu} a_{\lambda \mu k} b_{\lambda \mu \nu} \\
& =\langle\mathcal{A}, \mathcal{B}\rangle=\langle\mathcal{A}, \mathcal{B}\rangle_{1: 3}, & \text { (2-tensor) },
\end{array}
$$

We will refer to the first two as partial contractions. We will also have contracted products where the contractions are made in different modes from the two factors. For example, we write $\mathcal{C}=\langle\mathcal{A}, \mathcal{B}\rangle_{1 ; 2}$ when the contraction is made in the first mode of $\mathcal{A}$ with the second mode of $\mathcal{B}$. The entries of $\mathcal{C}$ are given by $c_{j k \mu \nu}=\sum_{\lambda} a_{\lambda j k} b_{\mu \lambda \nu}$. Similarly, we may have $D=\langle\mathcal{A}, \mathcal{B}\rangle_{1,3 ; 2,1}$, where the entries of $D$ are given by $d_{j \mu}=$ $\sum_{\lambda, \nu} a_{\lambda j \nu} b_{\nu \lambda \mu}$. In general, the subscripts in the contraction symbols, separated with a semicolon, indicate the modes of the first argument followed by the modes of the second argument that are contracted. For clarity in notation, when the contractions are made along the same modes in both arguments, only one set of subscripts is given. Observe also that we let the ordering of the modes in contracted tensor products be implicitly given in the summation. Thus given $\mathcal{A} \in \mathbb{R}^{l \times m \times n}$ and $\mathcal{B} \in \mathbb{R}^{l \times q \times r}$, then

$$
\mathcal{C}=\langle\mathcal{A}, \mathcal{B}\rangle_{1} \in \mathbb{R}^{m \times n \times q \times r} .
$$

In general, the modes of the product are given by the ordering of the noncontracted modes of the first argument followed by the ordering of the noncontracted modes of the second argument.

We will also use negative subscripts when the the contraction is made in all but a few modes. For 3-tensors we have

$$
\langle\mathcal{A}, \mathcal{B}\rangle_{2,3} \equiv\langle\mathcal{A}, \mathcal{B}\rangle_{-1}, \quad\langle\mathcal{A}, \mathcal{B}\rangle_{2} \equiv\langle\mathcal{A}, \mathcal{B}\rangle_{-(1,3)} .
$$

It is easily seen that the elements of the matrix $\langle\mathcal{A}, \mathcal{B}\rangle_{-1}$ are inner products of the mode- 1 slices of the two tensors. Similarly, the elements of the 4 -tensor $\langle\mathcal{A}, \mathcal{B}\rangle_{-(1,3)}$ are inner products of mode-2 fibers of the two tensors.

In contracted products there is no restriction that requires the two arguments to be tensors of same order. For example, with a 4 -tensor $\mathcal{A}$ and matrices (2-tensors) $F$ and $G$,

$$
\langle\mathcal{A}, F\rangle_{3,4 ; 1,2}=G, \quad \text { where } \quad g_{i j}=\sum_{\mu, \nu} a_{i j \mu \nu} f_{\mu \nu},
$$

defines a linear system of equations. This defines an operator $\mathbf{A}$ that maps matrices on matrices, i.e., $\mathbf{A}: X \rightarrow\langle\mathcal{A}, X\rangle_{1,2 ; 1,2}$. Then, the operator norm associated with the Frobenius "vector" norm is denoted

$$
\|\mathbf{A}\|_{2}=\max _{\|X\|=1}\|\mathbf{A}(X)\|
$$


The following lemma from [12] will be needed.

Lemma 2.1. Let the order $k$ tensors $\mathcal{B}$ and $\mathcal{C}$ and the matrix $Q$ be of conforming dimensions. Then

$$
\begin{aligned}
\left\langle\mathcal{B} \cdot(Q)_{i}, \mathcal{C}\right\rangle_{-i} & =Q^{\top}\langle\mathcal{B}, \mathcal{C}\rangle_{-i}=\left\langle Q,\langle\mathcal{B}, \mathcal{C}\rangle_{-i}\right\rangle_{1} \\
\left\langle\mathcal{B}, \mathcal{C} \cdot\left(Q^{\top}\right)_{i}\right\rangle_{-i} & =\langle\mathcal{B}, \mathcal{C}\rangle_{-i} Q^{\top}=\left\langle\langle\mathcal{B}, \mathcal{C}\rangle_{-i}, Q\right\rangle_{2}
\end{aligned}
$$

2.3. Multilinear rank. The multilinear rank of a third order tensor $\mathcal{A}$ is an integer triplet $(p, q, r)$ such that

$$
\begin{aligned}
& p=\operatorname{dim}\left(\operatorname{range}\left(A^{(1)}\right)\right)=\operatorname{rank}\left(A^{(1)}\right), \\
& q=\operatorname{dim}\left(\operatorname{range}\left(A^{(2)}\right)\right)=\operatorname{rank}\left(A^{(2)}\right), \\
& r=\operatorname{dim}\left(\operatorname{range}\left(A^{(3)}\right)\right)=\operatorname{rank}\left(A^{(3)}\right),
\end{aligned}
$$

where range $\left(A^{(i)}\right)=\left\{y \mid y=A^{(i)} x\right\}$ denotes the range space of $A^{(i)}$, which is the mode- $i$ matricization of $\mathcal{A}$, and $\operatorname{rank}\left(A^{(i)}\right)$ is the matrix rank. Multilinear rank is discussed in [8], as well as other rank concepts. In this paper we will deal only with multilinear rank, and we will use the notation $\operatorname{rank}-(p, q, r)$, and $\operatorname{rank}(\mathcal{A})=(p, q, r)$.

For matrices the rank is obtained via the SVD; see, e.g., [13, Chapter 2]. In exact arithmetic the multilinear rank can be computed using the higher order singular value decomposition (HOSVD) [5].

3. Best rank- $(p, q, r)$ approximation. Assume that we want to approximate in the norm (2.7) the tensor $\mathcal{A}$ by another tensor $\mathcal{B}$ of rank- $(p, q, r)$. Thus we want to solve

$$
\min _{\operatorname{rank}(\mathcal{B})=(p, q, r)}\|\mathcal{A}-\mathcal{B}\| .
$$

This problem is treated in [6]. In the matrix case, the solution of the corresponding problem is given by the truncated SVD. This is the Eckart-Young property [9]; a simple proof is given in [11, Theorem 6.7]. In view of the fact that the HOSVD "orders the mass" ${ }^{2}[5]$ of the tensor in a similar way as the SVD, one might think that a truncated HOSVD would give the solution of (3.1). However, this is not the case [6]. Some theoretical questions concerning the best rank- $(p, q, r)$ approximation problem are studied in [8]. In particular the following result is proved.

Proposition 3.1. Every order $k$ tensor $\mathcal{A}$ has a best approximation $\mathcal{B}$ with $\operatorname{rank}(\mathcal{B}) \leq\left(r_{1}, r_{2}, \ldots, r_{k}\right)$ for any specified $\left(r_{1}, r_{2}, \ldots, r_{k}\right)$.

In $[6,12]$ it is shown that the problem of solving $(3.1)$, i.e., making the residual as small as possible, is equivalent to determining $X, Y$, and $Z$, with orthonormal columns, so that

$$
\left\|\left(X^{\top}, Y^{\top}, Z^{\top}\right) \cdot \mathcal{A}\right\|=\|\mathcal{A} \cdot(X, Y, Z)\|
$$

is maximized. We thus define the objective function

$$
\Phi(X, Y, Z)=\frac{1}{2}\|\mathcal{A} \cdot(X, Y, Z)\|^{2}=\frac{1}{2} \sum_{i, j, k} f_{i j k}^{2}, \quad f_{i j k}=\sum_{\lambda, \mu, \nu} a_{\lambda \mu \nu} x_{\lambda i} y_{\mu j} z_{\nu k},
$$

where $x_{\lambda i}, y_{\mu j}$, and $z_{\nu k}$ are elements of $X, Y$, and $Z$, respectively.

\footnotetext{
${ }^{2}$ We here somewhat loosely use the word "mass" in the Frobenius norm sense.
} 
3.1. Best rank- $(p, q, r)$ approximation on a product of Grassmann manifolds. In [12] a Newton-Grassmann method is derived for solving the maximization problem on a product of Grassmann manifolds. We will give a brief sketch of the basic results. It follows from the invariance of the norm under orthogonal transformations that

$$
\Phi(X, Y, Z)=\Phi(X U, Y V, Z W)
$$

for orthogonal matrices $U \in \mathbb{R}^{p \times p}, V \in \mathbb{R}^{q \times q}$, and $W \in \mathbb{R}^{r \times r}$. This means that the problem of maximizing $\Phi$ under the orthogonality constraint is not yet well defined: it is overparameterized, and any straightforward constrained optimization method would have difficulties. It follows that the function $\Phi$ should be maximized not over matrices with orthonormal columns but over equivalence classes of such matrices. Denote the equivalence class of matrices that span the same subspace by

$$
[X]=\{X U \mid U \text { orthogonal }\} .
$$

This means that the maximization should be over the Grassmann manifold [10], or more precisely, over a product of three Grassmann manifolds. We will use $\operatorname{Gr}(l, p)$ to denote the Grassmann manifold of $p$ dimensional subspaces in $\mathbb{R}^{l}$, or equivalently the set of all equivalence classes of $l \times p$ orthonormal matrices. Any matrix $X \in \mathbb{R}^{l \times p}$ from $[X]$ may represent the entire equivalence class as a point on the manifold. We simply write $X \in \operatorname{Gr}(l, p)$. All statements in the following will be independent of the choice of a representative of the equivalence class.

The objective function $\Phi(X, Y, Z)$ depends on three matrices, and we will write $(X, Y, Z) \in \mathrm{Gr}^{3} \equiv \operatorname{Gr}(l, p) \times \operatorname{Gr}(m, q) \times \operatorname{Gr}(n, r)$ to mean $X \in \operatorname{Gr}(l, p), Y \in \operatorname{Gr}(m, q)$, and $Z \in \operatorname{Gr}(n, r)$. Since we will optimize over $\mathrm{Gr}^{3}$, which is not a vector space, we will need to make statements on optimality and perturbations in terms of its tangent space $\mathbb{T}^{3}$; see, e.g., [20, p. 307].

The tangent space at a point $X \in \operatorname{Gr}(l, p)$ is [10]

$$
\mathbb{T}_{X}=\left\{\Delta \in \mathbb{R}^{l \times p} \mid X^{\top} \Delta=0\right\} .
$$

The product of tangent spaces at $(X, Y, Z) \in \mathrm{Gr}^{3}$ will be denoted $\mathbb{T}^{3} \equiv \mathbb{T}_{X} \times \mathbb{T}_{Y} \times$ $\mathbb{T}_{Z}$. The canonical inner product between two tangents $\Delta_{x}$ and $\Gamma_{x}$ in $\mathbb{T}_{X}$ is defined $\left\langle\Delta_{x}, \Gamma_{x}\right\rangle=\operatorname{tr}\left(\Delta_{x}^{\top} \Gamma_{x}\right)$. The inner product between $\bar{\Delta}=\left(\Delta_{x}, \Delta_{y}, \Delta_{z}\right) \in \mathbb{T}^{3}$ and $\bar{\Gamma}=\left(\Gamma_{x}, \Gamma_{y}, \Gamma_{z}\right) \in \mathbb{T}^{3}$ is

$$
\langle\bar{\Delta}, \bar{\Gamma}\rangle=\left\langle\Delta_{x}, \Gamma_{x}\right\rangle+\left\langle\Delta_{y}, \Gamma_{y}\right\rangle+\left\langle\Delta_{z}, \Gamma_{z}\right\rangle .
$$

3.2. Gradient and Hessian on the product manifold. The constrained optimization problem we consider is

$$
\max _{(X, Y, Z) \in \mathrm{Gr}^{3}} \Phi(X, Y, Z), \quad \text { where } \quad \Phi(X, Y, Z)=\frac{1}{2}\|\mathcal{A} \cdot(X, Y, Z)\|^{2} .
$$

We will now state the Grassmann gradient and the Grassmann Hessian of $\Phi$. For more details, see $[12,26]$. The Grassmann gradient of $\Phi$ is an object in $\mathbb{T}^{3}$,

$$
\nabla \Phi=\left(\Pi_{X} \Phi_{x}, \Pi_{Y} \Phi_{y}, \Pi_{Z} \Phi_{z}\right) \in \mathbb{T}^{3},
$$


where

$$
\begin{aligned}
& \Pi_{X} \Phi_{x}=\left\langle\mathcal{A} \cdot\left(\Pi_{X}, Y, Z\right), \mathcal{F}\right\rangle_{-1} \in \mathbb{T}_{X}, \quad \Pi_{X}=I-X X^{\top}, \\
& \Pi_{Y} \Phi_{y}=\left\langle\mathcal{A} \cdot\left(X, \Pi_{Y}, Z\right), \mathcal{F}\right\rangle_{-2} \in \mathbb{T}_{Y}, \quad \Pi_{Y}=I-Y Y^{\top}, \\
& \Pi_{Z} \Phi_{z}=\left\langle\mathcal{A} \cdot\left(X, Y, \Pi_{Z}\right), \mathcal{F}\right\rangle_{-3} \in \mathbb{T}_{Z}, \quad \Pi_{Z}=I-Z Z^{\top},
\end{aligned}
$$

where $\Phi_{x}=\partial \Phi / \partial X, \Phi_{y}=\partial \Phi / \partial Y$, and $\Phi_{z}=\partial \Phi / \partial Z$ are partial derivatives of $\Phi$ with respect to entries of $X, Y$, and $Z$, respectively, and $\mathcal{F}=\mathcal{A} \cdot(X, Y, Z)$. After some manipulation we get

$$
\begin{aligned}
\Pi_{X} \Phi_{x} & =\langle\mathcal{A} \cdot(I, Y, Z), \mathcal{A} \cdot(I, Y, Z)\rangle_{-1} X-X\langle\mathcal{F}, \mathcal{F}\rangle_{-1}, \\
\Pi_{Y} \Phi_{y} & =\langle\mathcal{A} \cdot(X, I, Z), \mathcal{A} \cdot(X, I, Z)\rangle_{-2} Y-Y\langle\mathcal{F}, \mathcal{F}\rangle_{-2}, \\
\Pi_{Y} \Phi_{z} & =\langle\mathcal{A} \cdot(X, Y, I), \mathcal{A} \cdot(X, Y, I)\rangle_{-3} Z-Z\langle\mathcal{F}, \mathcal{F}\rangle_{-3} .
\end{aligned}
$$

Deriving the Grassmann Hessian of $\Phi$ is somewhat more involved. It can be considered as a linear (and self-adjoint) operator $\mathbf{H}: \mathbb{T}^{3} \rightarrow \mathbb{T}^{3}$. With $\bar{\Delta}=\left(\Delta_{x}, \Delta_{y}, \Delta_{z}\right) \in$ $\mathbb{T}^{3}$ the action of the Grassmann Hessian is given by $\mathbf{H}(\bar{\Delta})=\left(\Gamma_{x}, \Gamma_{y}, \Gamma_{z}\right)$, where

$$
\begin{aligned}
\Gamma_{x} & =\mathbf{H}_{x x}\left(\Delta_{x}\right)+\mathbf{H}_{x y}\left(\Delta_{y}\right)+\mathbf{H}_{x z}\left(\Delta_{z}\right), \\
\Gamma_{y} & =\mathbf{H}_{y x}\left(\Delta_{x}\right)+\mathbf{H}_{y y}\left(\Delta_{y}\right)+\mathbf{H}_{y z}\left(\Delta_{z}\right), \\
\Gamma_{z} & =\mathbf{H}_{z x}\left(\Delta_{x}\right)+\mathbf{H}_{z y}\left(\Delta_{y}\right)+\mathbf{H}_{z z}\left(\Delta_{z}\right),
\end{aligned}
$$

and each $\mathbf{H}_{* *}(\cdot)$ is a linear operator to be further specified below. The diagonal blocks ${ }^{3}$ are

$$
\begin{array}{ll}
\mathbf{H}_{x x}\left(\Delta_{x}\right)=\left\langle\mathcal{F}^{x}, \mathcal{F}^{x}\right\rangle_{-1} \Delta_{x}-\Delta_{x}\langle\mathcal{F}, \mathcal{F}\rangle_{-1}, & \mathcal{F}^{x}=\mathcal{A} \cdot\left(\Pi_{x}, Y, Z\right), \\
\mathbf{H}_{y y}\left(\Delta_{y}\right)=\left\langle\mathcal{F}^{y}, \mathcal{F}^{y}\right\rangle_{-2} \Delta_{y}-\Delta_{y}\langle\mathcal{F}, \mathcal{F}\rangle_{-2}, & \mathcal{F}^{y}=\mathcal{A} \cdot\left(X, \Pi_{y}, Z\right), \\
\mathbf{H}_{z z}\left(\Delta_{z}\right)=\left\langle\mathcal{F}^{z}, \mathcal{F}^{z}\right\rangle_{-3} \Delta_{z}-\Delta_{z}\langle\mathcal{F}, \mathcal{F}\rangle_{-3}, & \mathcal{F}^{z}=\mathcal{A} \cdot\left(X, Y, \Pi_{z}\right),
\end{array}
$$

where $\mathcal{F}=\mathcal{A} \cdot(X, Y, Z)$. Since $\mathbf{H}$ is self-adjoint we give only the blocks "above the diagonal,"

$$
\begin{aligned}
& \mathbf{H}_{x y}\left(\Delta_{y}\right)=\left\langle\left\langle\mathcal{F}^{x y}, \mathcal{F}\right\rangle_{-(1,2)}, \Delta_{y}\right\rangle_{2,4 ; 1,2}+\left\langle\left\langle\mathcal{F}^{x}, \mathcal{F}^{y}\right\rangle_{-(1,2)}, \Delta_{y}\right\rangle_{4,2 ; 1,2}, \\
& \mathbf{H}_{x z}\left(\Delta_{z}\right)=\left\langle\left\langle\mathcal{F}^{x z}, \mathcal{F}\right\rangle_{-(1,3)}, \Delta_{z}\right\rangle_{2,4 ; 1,2}+\left\langle\left\langle\mathcal{F}^{x}, \mathcal{F}^{z}\right\rangle_{-(1,3)}, \Delta_{z}\right\rangle_{4,2 ; 1,2}, \\
& \mathbf{H}_{y z}\left(\Delta_{z}\right)=\left\langle\left\langle\mathcal{F}^{y z}, \mathcal{F}\right\rangle_{-(2,3)}, \Delta_{z}\right\rangle_{2,4 ; 1,2}+\left\langle\left\langle\mathcal{F}^{y}, \mathcal{F}^{z}\right\rangle_{-(2,3)}, \Delta_{z}\right\rangle_{4,2 ; 1,2},
\end{aligned}
$$

where we have also introduced $\mathcal{F}^{x y}=\mathcal{A} \cdot\left(\Pi_{x}, \Pi_{y}, Z\right), \mathcal{F}^{x z}=\mathcal{A} \cdot\left(\Pi_{x}, Y, \Pi_{z}\right)$, and $\mathcal{F}^{y z}=\mathcal{A} \cdot\left(X, \Pi_{y}, \Pi_{z}\right)$. Observe that diagonal blocks (3.13)-(3.15) are Sylvester operators, and the off-diagonal blocks (3.16)-(3.18) have the form of 4-tensors acting on matrices, e.g., $\left\langle\mathcal{F}^{x y}, \mathcal{F}\right\rangle_{-(1,2)}$ is a 4-tensor that acts on the matrix $\Delta_{y}$ through the contraction $\left\langle\left\langle\mathcal{F}^{x y}, \mathcal{F}\right\rangle_{-(1,2)}, \Delta_{y}\right\rangle_{2,4 ; 1,2}$.

3.3. Gradient and Hessian operator in local coordinates. The expressions for the gradient (3.7) and Hessian (3.12) are given in global coordinates, since they are represented as objects in the ambient Euclidean space. In the study of the stationary points for the function $\Phi(X, Y, Z)$ we prefer to use local coordinate expressions for

\footnotetext{
${ }^{3}$ In analogy with matrices we will refer to the operators $\mathbf{H}_{x x}, \mathbf{H}_{y y}$, and $\mathbf{H}_{z z}$ as diagonal blocks, and $\mathbf{H}_{x y}$, etc. as off-diagonal blocks. The term diagonal operator will be used for operators that are diagonal in a structural sense, i.e., generalizations of diagonal matrices.
}

Copyright (C) by SIAM. Unauthorized reproduction of this article is prohibited. 
the gradient and the Hessian. The analysis in sections 4 and 5 may also be done using global coordinate expressions for the involved quantities, but that approach does not reveal the structure of the problem that we want to uncover. It is also much more difficult to make comparisons to the matrix case. We will use the following notation. Given a matrix $X$ with orthonormal columns, $X_{\perp}$ denotes a matrix such that $\left[\begin{array}{l}X \\ X_{\perp}\end{array}\right]$ is orthogonal (and, of course, square). If $X$ represents a point on the manifold, then from (3.5) we see that every element of $\mathbb{T}_{X}$ can be written $X_{\perp} D_{x}$, for some matrix of coordinates $D_{x}$. It turns out that this is a representation with the correct number of degrees of freedom; see [10]. More details are also given in $[12,26]$.

It is shown in [12] that the local coordinate expression for the Grassmann gradient is

$$
\nabla \widehat{\Phi}=\left(X_{\perp}^{\top} \Phi_{x}, Y_{\perp}^{\top} \Phi_{y}, Z_{\perp}^{\top} \Phi_{z}\right)=\left(\left\langle\mathcal{F}_{\perp}^{x}, \mathcal{F}\right\rangle_{-1},\left\langle\mathcal{F}_{\perp}^{y}, \mathcal{F}\right\rangle_{-2},\left\langle\mathcal{F}_{\perp}^{z}, \mathcal{F}\right\rangle_{-3}\right)
$$

where $\mathcal{F}_{\perp}^{x}=\mathcal{A} \cdot\left(X_{\perp}, Y, Z\right), \mathcal{F}_{\perp}^{y}=\mathcal{A} \cdot\left(X, Y_{\perp}, Z\right)$, and $\mathcal{F}_{\perp}^{z}=\mathcal{A} \cdot\left(X, Y, Z_{\perp}\right)$.

The global coordinate Hessian $\mathbf{H}$ acts on $\bar{\Delta}=\left(\Delta_{x}, \Delta_{y}, \Delta_{z}\right) \in \mathbb{T}^{3}$. Using local coordinates for the tangents

$$
\begin{array}{ll}
\Delta_{x}=X_{\perp} D_{x}, & D_{x} \in \mathbb{R}^{(l-p) \times p}, \\
\Delta_{y}=Y_{\perp} D_{y}, & D_{y} \in \mathbb{R}^{(m-q) \times q}, \\
\Delta_{z}=Z_{\perp} D_{z}, & D_{x} \in \mathbb{R}^{(n-r) \times r},
\end{array}
$$

we can write the Hessian as a linear operator acting on $\bar{D}=\left(D_{x}, D_{y}, D_{z}\right)$. The local coordinate Grassmann Hessian becomes $\widehat{\mathbf{H}}(\bar{D})=\left(G_{x}, G_{y}, G_{z}\right)$, where

$$
\begin{aligned}
G_{x} & =\widehat{\mathbf{H}}_{x x}\left(D_{x}\right)+\widehat{\mathbf{H}}_{x y}\left(D_{y}\right)+\widehat{\mathbf{H}}_{x z}\left(D_{z}\right), \\
G_{y} & =\widehat{\mathbf{H}}_{y x}\left(D_{x}\right)+\widehat{\mathbf{H}}_{y y}\left(D_{y}\right)+\widehat{\mathbf{H}}_{y z}\left(D_{z}\right), \\
G_{z} & =\widehat{\mathbf{H}}_{z x}\left(D_{x}\right)+\widehat{\mathbf{H}}_{z y}\left(D_{y}\right)+\widehat{\mathbf{H}}_{z z}\left(D_{z}\right),
\end{aligned}
$$

and, again, each $\widehat{\mathbf{H}}_{* *}(\cdot)$ is a linear operator. The diagonal blocks are

$$
\begin{aligned}
\widehat{\mathbf{H}}_{x x}\left(D_{x}\right) & =\left\langle\mathcal{F}_{\perp}^{x}, \mathcal{F}_{\perp}^{x}\right\rangle_{-1} D_{x}-D_{x}\langle\mathcal{F}, \mathcal{F}\rangle_{-1}, \\
\widehat{\mathbf{H}}_{y y}\left(D_{y}\right) & =\left\langle\mathcal{F}_{\perp}^{y}, \mathcal{F}_{\perp}^{y}\right\rangle_{-2} D_{y}-D_{y}\langle\mathcal{F}, \mathcal{F}\rangle_{-2}, \\
\widehat{\mathbf{H}}_{z z}\left(D_{z}\right) & =\left\langle\mathcal{F}_{\perp}^{z}, \mathcal{F}_{\perp}^{z}\right\rangle_{-3} D_{z}-D_{z}\langle\mathcal{F}, \mathcal{F}\rangle_{-3},
\end{aligned}
$$

where $\mathcal{F}_{\perp}^{x}, \mathcal{F}_{\perp}^{y}$, and $\mathcal{F}_{\perp}^{z}$ are introduced in (3.19). The blocks "above the diagonal" are

$$
\begin{aligned}
\widehat{\mathbf{H}}_{x y}\left(D_{y}\right) & =\left\langle\left\langle\mathcal{F}_{\perp}^{x y}, \mathcal{F}\right\rangle_{-(1,2)}, D_{y}\right\rangle_{2,4 ; 1,2}+\left\langle\left\langle\mathcal{F}_{\perp}^{x}, \mathcal{F}_{\perp}^{y}\right\rangle_{-(1,2)}, D_{y}\right\rangle_{4,2 ; 1,2} \\
\widehat{\mathbf{H}}_{x z}\left(D_{z}\right) & =\left\langle\left\langle\mathcal{F}_{\perp}^{x z}, \mathcal{F}\right\rangle_{-(1,3)}, D_{z}\right\rangle_{2,4 ; 1,2}+\left\langle\left\langle\mathcal{F}_{\perp}^{x}, \mathcal{F}_{\perp}^{z}\right\rangle_{-(1,3)}, D_{z}\right\rangle_{4,2 ; 1,2} \\
\widehat{\mathbf{H}}_{y z}\left(D_{z}\right) & =\left\langle\left\langle\mathcal{F}_{\perp}^{y z}, \mathcal{F}\right\rangle_{-(2,3)}, D_{z}\right\rangle_{2,4 ; 1,2}+\left\langle\left\langle\mathcal{F}_{\perp}^{y}, \mathcal{F}_{\perp}^{z}\right\rangle_{-(2,3)}, D_{z}\right\rangle_{4,2 ; 1,2}
\end{aligned}
$$

where $\mathcal{F}_{\perp}^{x y}=\mathcal{A} \cdot\left(X_{\perp}, Y_{\perp}, Z\right), \mathcal{F}_{\perp}^{x z}=\mathcal{A} \cdot\left(X_{\perp}, Y, Z_{\perp}\right)$, and $\mathcal{F}_{\perp}^{y z}=\mathcal{A} \cdot\left(X, Y_{\perp}, Z_{\perp}\right)$.

Since $\widehat{\mathbf{H}}$ is a self-adjoint operator acting on a real, finite-dimensional vector space, it can be represented by a symmetric matrix; it has real eigenvalues and a full set of orthogonal eigenvectors. However, unfolding the Hessian as a matrix makes it more complicated to recognize its structure, and therefore we shall keep it in operator form in what follows.

Copyright (c) by SIAM. Unauthorized reproduction of this article is prohibited. 
It is well known $[6,15]$ that, in general, the objective function (3.6) is not concave. In fact it is easy to construct nonconcave examples using the coordinate representation of the Hessian.

Proposition 3.2. Let $\mathcal{A}$ be a tensor of order 3 or higher. The maximization problem (3.6) can have local maxima that are not a global maximum.

To illustrate Proposition 3.2, consider the $2 \times 2 \times 2$ tensor

$$
\mathcal{A}(:,:, 1)=\left[\begin{array}{ll}
1 & 0 \\
0 & 0
\end{array}\right], \quad \mathcal{A}(:,:, 2)=\left[\begin{array}{cc}
0 & 0 \\
0 & 100
\end{array}\right] .
$$

It is straightforward to verify (by computing the gradient and Hessian) that the point $x=y=z=\left[\begin{array}{ll}1 & 0\end{array}\right]^{\top}$ is a local maximum, whereas the global maximum is given by $x=y=z=\left[\begin{array}{ll}0 & 1\end{array}\right]^{\top}$.

Example 3.3. In order to see in what way the tensor case is different from the matrix case, we now consider the latter. In particular, we will demonstrate that for a matrix (a second order tensor) Proposition 3.2 is not valid.

Let $\mathrm{Gr}^{2}=\operatorname{Gr}(n, r) \times \operatorname{Gr}(n, r)$ be the product of two Grassmann manifolds. Given a diagonal matrix $\Sigma \in \mathbb{R}^{n \times n}$, where the diagonal elements $\sigma_{i}$ are assumed to be nonnegative, ${ }^{4}$ we want to maximize

$$
\max _{(X, Y) \in \mathrm{Gr}^{2}}\left\|X^{\top} \Sigma Y\right\|
$$

Let $\left[i_{1}, i_{2}, \ldots, i_{n}\right]$ be a permutation of the set $\{1,2, \ldots, n\}$. Choose

$$
X=Y=\left[\begin{array}{llll}
e_{i_{1}} & e_{i_{2}} & \cdots & e_{i_{r}}
\end{array}\right], \quad X_{\perp}=Y_{\perp}=\left[\begin{array}{llll}
e_{i_{r+1}} & e_{i_{r+2}} & \cdots & e_{i_{n}}
\end{array}\right],
$$

where the column vectors are standard unit basis vectors. Then

$$
\left[\begin{array}{ll}
X & X_{\perp}
\end{array}\right]^{\top} \Sigma\left[\begin{array}{ll}
Y & Y_{\perp}
\end{array}\right]=\left[\begin{array}{cc}
\Sigma_{1} & 0 \\
0 & \Sigma_{2}
\end{array}\right]
$$

where $\Sigma_{1}=\operatorname{diag}\left(\sigma_{i_{1}}, \ldots, \sigma_{i_{r}}\right)$ and $\Sigma_{2}=\operatorname{diag}\left(\sigma_{i_{r+1}}, \ldots, \sigma_{i_{n}}\right)$. In tensor notation, treating the matrix $\Sigma$ as a tensor in $\mathbb{R}^{n \times n}$, we have

$$
\left[\begin{array}{cc}
F & F_{\perp}^{y} \\
F_{\perp}^{x} & F_{\perp}^{x y}
\end{array}\right]=\left[\begin{array}{cc}
\Sigma \cdot(X, Y) & \Sigma \cdot\left(X, Y_{\perp}\right) \\
\Sigma \cdot\left(X_{\perp}, Y\right) & \Sigma \cdot\left(X_{\perp}, Y_{\perp}\right)
\end{array}\right]=\left[\begin{array}{cc}
\Sigma_{1} & 0 \\
0 & \Sigma_{2}
\end{array}\right] .
$$

The operator $\widehat{\mathbf{H}}_{x x}$ acting on $D_{x}$ becomes

$$
\widehat{\mathbf{H}}_{x x}\left(D_{x}\right)=-D_{x}\langle F, F\rangle_{-1}=-D_{x} \Sigma_{1}^{2},
$$

since $F_{\perp}^{x}=0$. Similarly, $\widehat{\mathbf{H}}_{y y}\left(D_{y}\right)=-D_{y} \Sigma_{1}^{2}$. Due to the diagonality of $F_{\perp}^{x y}=\Sigma_{2}$ and $F=\Sigma_{1}$, the $(\mu, \nu)$ element of $\widehat{\mathbf{H}}_{x y}\left(D_{y}\right)$ is

$$
\left(\widehat{\mathbf{H}}_{x y}\left(D_{y}\right)\right)_{\mu, \nu}=\left(\left\langle\left\langle F_{\perp}^{x y}, F\right\rangle_{-(1,2)}, D_{y}\right\rangle_{2,4 ; 1,2}\right)_{\mu, \nu}=\sigma_{i_{\mu}} \sigma_{i_{\nu}}\left(D_{y}\right)_{\mu, \nu},
$$

where $1 \leq \mu \leq r$ and $r+1 \leq \nu \leq n$; (note that the $-(1,2)$ contraction is vacuous, since both $F_{\perp}^{x y}$ and $F$ are matrices). Thus $\widehat{\mathbf{H}}_{x y}\left(D_{y}\right)=\Sigma_{1} D_{y} \Sigma_{2}$. The Hessian operator can now be written

$$
\left[\begin{array}{c}
\widehat{\mathbf{H}}_{x x}\left(D_{x}\right)+\widehat{\mathbf{H}}_{x y}\left(D_{y}\right) \\
\widehat{\mathbf{H}}_{y x}\left(D_{x}\right)+\widehat{\mathbf{H}}_{y y}\left(D_{y}\right)
\end{array}\right]=\left[\begin{array}{c}
-D_{x} \Sigma_{1}^{2}+\Sigma_{1} D_{y} \Sigma_{2} \\
\Sigma_{1} D_{x} \Sigma_{2}-D_{y} \Sigma_{2}^{2}
\end{array}\right] .
$$

\footnotetext{
${ }^{4}$ It is no restriction, of course, to assume diagonality and nonnegativity.
} 
From the derivations above we see that we cannot modify the matrix $\Sigma$ in any position, diagonal or off-diagonal, without changing the value of $\widehat{\mathbf{H}}(D)$. This is in contrast to the tensor case, where the $\mathcal{A} \cdot\left(X_{\perp}, Y_{\perp}, Z_{\perp}\right)$ part of the transformed tensor does not occur in the gradient or Hessian, and thus can be changed without affecting the stationarity of a point $(X, Y, Z)$.

For later use, we rewrite the Hessian operator in matrix-vector form,

$$
\left[\begin{array}{cc}
-\Sigma_{1}^{2} \otimes I & \Sigma_{2} \otimes \Sigma_{1} \\
\Sigma_{2} \otimes \Sigma_{1} & -\Sigma_{1}^{2} \otimes I
\end{array}\right]\left[\begin{array}{c}
\operatorname{vec}\left(D_{x}\right) \\
\operatorname{vec}\left(D_{y}\right)
\end{array}\right]
$$

where $\otimes$ denotes the Kronecker product of matrices, and $\operatorname{vec}(D)$ is a vector consisting of the stacked column vectors of a matrix $D$.

3.4. Interpretation of operators in the Hessian. The Hessian operator $\widehat{\mathbf{H}}$ consists of partial contractions involving the tensors

$$
\begin{array}{llll}
\mathcal{A} \cdot(X, Y, Z), & \mathcal{A} \cdot\left(X_{\perp}, Y, Z\right), & \mathcal{A} \cdot\left(X, Y_{\perp}, Z\right), & \mathcal{A} \cdot\left(X, Y, Z_{\perp}\right), \\
\mathcal{A} \cdot\left(X, Y_{\perp}, Z_{\perp}\right), & \mathcal{A} \cdot\left(X_{\perp}, Y, Z_{\perp}\right), & \mathcal{A} \cdot\left(X_{\perp}, Y_{\perp}, Z\right) .
\end{array}
$$

These are blocks of the tensor $\widehat{\mathcal{A}}=\mathcal{A} \cdot\left(\left[\begin{array}{ll}X & X_{\perp}\end{array}\right],\left[\begin{array}{ll}Y & Y_{\perp}\end{array}\right],\left[\begin{array}{ll}Z & Z_{\perp}\end{array}\right]\right)$. The only block in $\widehat{\mathcal{A}}$ that does not occur in $\widehat{\mathcal{H}}$ is $\mathcal{A} \cdot\left(X_{\perp}, Y_{\perp}, Z_{\perp}\right)$. $\widehat{\mathcal{A}}$ is illustrated in Figure 3.1. Note that $\widehat{\mathcal{A}}$ is the tensor that is obtained if a change of coordinate system is made in all three modes, with the matrices $\left[X X_{\perp}\right],\left[\begin{array}{ll}Y & Y_{\perp}\end{array}\right]$, and $\left[\begin{array}{ll}Z & Z_{\perp}\end{array}\right]$, respectively. Making the analogy with the SVD, we see that $\widehat{\mathcal{A}}$ corresponds to the diagonal matrix $\Sigma$. Unlike the SVD, this transformed tensor is not sparse. However, later in section 4, we will see that if $(X, Y, Z)$ is a local maximum, then $\widehat{\mathcal{A}}$ satisfies certain orthogonality and ordering relations.

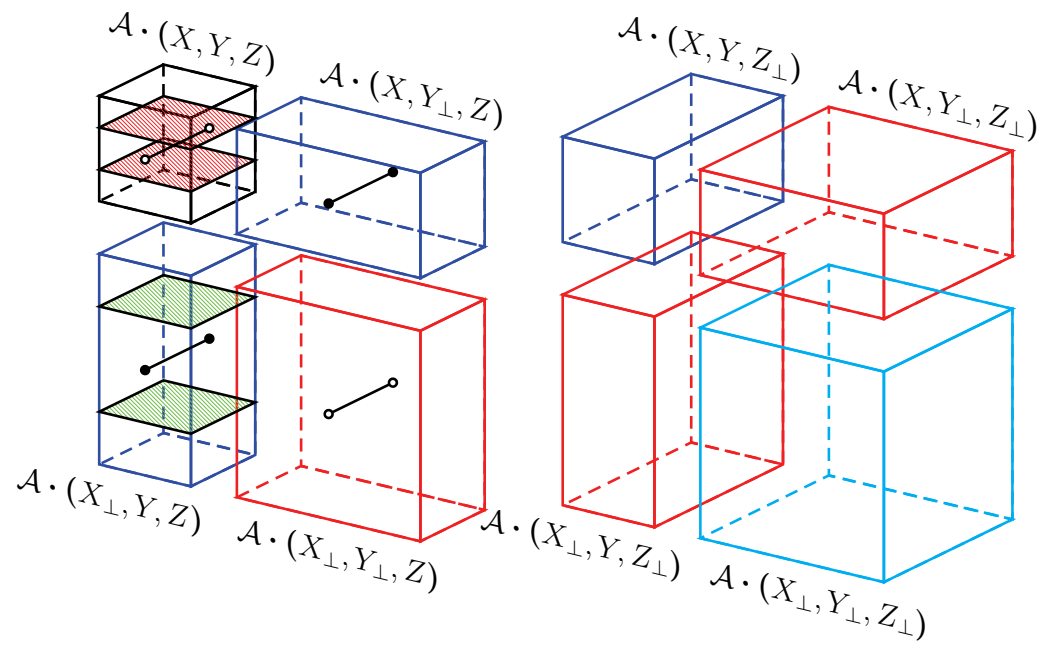

FIG. 3.1. Illustration of the partial contractions in the Hessian. For better visibility we have slid the backward part of the the tensor $\widehat{\mathcal{A}}$ to the right.

The partial contractions $\langle\cdot, \cdot\rangle_{-i}$ in $(3.22)$ are matrices whose elements are inner products between the slices in a subtensor. In Figure 3.1 we illustrate the inner products in $\widehat{\mathbf{H}}_{x x}$, which consists of the contractions $\left\langle\mathcal{F}_{\perp}^{x}, \mathcal{F}_{\perp}^{x}\right\rangle_{-1}$ and $\langle\mathcal{F}, \mathcal{F}\rangle_{-1}$. In the first contraction we have inner products between mode- 1 slices in $\mathcal{A} \cdot\left(X_{\perp}, Y, Z\right)$, 
and in the second contraction we have inner products between mode- 1 slices in $\mathcal{A}$. $(X, Y, Z)$; see the $(1,1,1)$ and $(2,1,1)$ blocks in Figure 3.1. In the off-diagonal blocks the inner products are between fibers in subtensors. For instance, in $\widehat{\mathbf{H}}_{x y}$ the inner products in $\left\langle\mathcal{F}_{\perp}^{x y}, \mathcal{F}\right\rangle_{-(1,2)}$ are between fibers, illustrated with the $\multimap \longrightarrow$ symbol, from $\mathcal{A} \cdot\left(X_{\perp}, Y_{\perp}, Z\right)$ and $\mathcal{A} \cdot(X, Y, Z)$. Similarly the elements of $\left\langle\mathcal{F}_{\perp}^{x}, \mathcal{F}_{\perp}^{y}\right\rangle_{-(1,2)}$ are inner products between the $\bullet$ fibers from $\mathcal{A} \cdot\left(X_{\perp}, Y, Z\right)$ and $\mathcal{A} \cdot\left(X, Y_{\perp}, Z\right)$.

4. Optimality conditions. By studying the conditions for optimality we can draw conclusion concerning some theoretical properties of the solution of the best multilinear rank tensor approximation problem. We assume that $(X, Y, Z)$ is a stationary point, i.e., a point on $\mathrm{Gr}^{3}$ such that the Grassmann gradient (3.19) is equal to zero. More precisely, $(X, Y, Z)$ is a representative of the equivalence class $([X],[Y],[Z])$, which is a point on the manifold. Then the rank- $(p, q, r)$ approximation is

$$
\mathcal{A} \approx(X, Y, Z) \cdot \mathcal{F}
$$

where the core is $\mathcal{F}=\mathcal{A} \cdot(X, Y, Z)$.

4.1. First order conditions. At a given stationary point $(X, Y, Z)$ the Grassmann gradient is equal to zero. Using the Grassmann gradient in global coordinates from (3.11), we have

$$
\begin{aligned}
& \langle\mathcal{A} \cdot(I, Y, Z), \mathcal{A} \cdot(I, Y, Z)\rangle_{-1} X-X\langle\mathcal{F}, \mathcal{F}\rangle_{-1}=0 \\
& \langle\mathcal{A} \cdot(X, I, Z), \mathcal{A} \cdot(X, I, Z)\rangle_{-2} Y-Y\langle\mathcal{F}, \mathcal{F}\rangle_{-2}=0, \\
& \langle\mathcal{A} \cdot(X, Y, I), \mathcal{A} \cdot(X, Y, I)\rangle_{-3} Z-Z\langle\mathcal{F}, \mathcal{F}\rangle_{-3}=0
\end{aligned}
$$

where $\mathcal{F}=\mathcal{A} \cdot(X, Y, Z)$. Using the local coordinate expression for the Grassmann gradient we get the following result.

Proposition 4.1. Let $(X, Y, Z)$ be a stationary point. Then the following orthogonality relations hold:

$$
\begin{aligned}
& \left\langle\mathcal{A} \cdot\left(X_{\perp}, Y, Z\right), \mathcal{A} \cdot(X, Y, Z)\right\rangle_{-1}=0, \\
& \left\langle\mathcal{A} \cdot\left(X, Y_{\perp}, Z\right), \mathcal{A} \cdot(X, Y, Z)\right\rangle_{-2}=0, \\
& \left\langle\mathcal{A} \cdot\left(X, Y, Z_{\perp}\right), \mathcal{A} \cdot(X, Y, Z)\right\rangle_{-3}=0 .
\end{aligned}
$$

Proof. These equations are simply the gradient in local coordinates (see (3.19)) and as such they are also zero at a stationary point.

Referring to Figure 3.1, (4.4) implies that the mode-1 slices of $\mathcal{F}=\mathcal{A} \cdot(X, Y, Z)$ (the upper left-most block) are orthogonal to the mode-1 slices of $\mathcal{F}_{\perp}^{x}=\mathcal{A} \cdot\left(X_{\perp}, Y, Z\right)$ (the lower left-most block). The other two equations can be interpreted analogously.

The SVD property of diagonalizing the matrix does not carry over to tensors and is replaced by this slicewise orthogonality. Furthermore, partitioning the SVD

$$
A=U \Sigma V^{\top}=\left[U_{1} U_{2}\right]\left[\begin{array}{cc}
\Sigma_{1} & 0 \\
0 & \Sigma_{2}
\end{array}\right]\left[\begin{array}{l}
V_{1}^{\top} \\
V_{2}^{\top}
\end{array}\right]
$$

we can write the the SVD equations as

$$
A V_{1}=\left[\begin{array}{ll}
U_{1} & U_{2}
\end{array}\right]\left[\begin{array}{c}
\Sigma_{1} \\
0
\end{array}\right], \quad A^{\top} U_{1}=\left[\begin{array}{ll}
V_{1} & V_{2}
\end{array}\right]\left[\begin{array}{c}
\Sigma_{1} \\
0
\end{array}\right] .
$$

Copyright $@$ by SIAM. Unauthorized reproduction of this article is prohibited. 
We now derive corresponding equations for the tensor case. Introducing the orthogonal matrix $\left[\begin{array}{ll}X & X_{\perp}\end{array}\right]$ and the identity $I=\left[\begin{array}{ll}X & X_{\perp}\end{array}\right]\left[\begin{array}{ll}X & X_{\perp}\end{array}\right]^{\top}$ we may write

$$
\begin{aligned}
\mathcal{A} \cdot(Y, Z)_{2,3} & =\mathcal{A} \cdot\left(\left[\begin{array}{ll}
{[X} & X_{\perp}
\end{array}\right]\left[X X_{\perp}\right]^{\top}, Y, Z\right)=\left(\left[X X_{\perp}\right]\right)_{1} \cdot\left[\begin{array}{c}
\mathcal{A} \cdot(X, Y, Z) \\
\mathcal{A} \cdot\left(X_{\perp}, Y, Z\right)
\end{array}\right] \\
& =(X)_{1} \cdot \mathcal{F}+\left(X_{\perp}\right)_{1} \cdot \mathcal{F}_{\perp}^{x}
\end{aligned}
$$

Unlike the SVD equation we cannot make the $\mathcal{F}_{\perp}^{x}$ block in (4.8) equal to zero, but from Proposition 4.1 we see that the slices of the tensors $\mathcal{A} \cdot(X, Y, Z)$ and $\mathcal{A} \cdot\left(X_{\perp}, Y, Z\right)$ are orthogonal in the sense of the mode- 1 contraction. An alternative presentation of this orthogonality would be to verify that the mode-1 matricization $A_{x}^{(1)}$ has orthogonal rows. We have the following result.

Proposition 4.2. Let $(X, Y, Z)$ be a stationary point, and let $\mathcal{F}=\mathcal{A} \cdot(X, Y, Z)$. Then we have

$$
\begin{aligned}
\mathcal{A} \cdot(Y, Z)_{2,3} & =(X)_{1} \cdot \mathcal{F}+\left(X_{\perp}\right)_{1} \cdot \mathcal{F}_{\perp}^{x} \\
& =(X)_{1} \cdot(\mathcal{A} \cdot(X, Y, Z))+\left(X_{\perp}\right)_{1} \cdot\left(\mathcal{A} \cdot\left(X_{\perp}, Y, Z\right)\right),
\end{aligned}
$$

where the slices of the tensors on the right-hand side are orthogonal in the sense of the contraction $\left\langle\mathcal{F}_{\perp}^{x}, \mathcal{F}\right\rangle_{-1}=0$.

The corresponding statements hold for the other modes:

$$
\mathcal{A} \cdot(X, Z)_{1,3}=(Y)_{2} \cdot \mathcal{F}+\left(Y_{\perp}\right)_{2} \cdot \mathcal{F}_{\perp}^{y}
$$

where $\left\langle\mathcal{F}_{\perp}^{y}, \mathcal{F}\right\rangle_{-2}=0$, and

$$
\mathcal{A} \cdot(X, Y)_{1,2}=(Z)_{3} \cdot \mathcal{F}+\left(Z_{\perp}\right)_{3} \cdot \mathcal{F}_{\perp}^{z}
$$

where $\left\langle\mathcal{F}_{\perp}^{z}, \mathcal{F}\right\rangle_{-3}=0$.

One may refer to $\mathcal{F}=\mathcal{A} \cdot(X, Y, Z)$ as the Rayleigh quotient tensor in analogy to the matrix case. It has the same optimal residual property as with matrices. The proof is a straightforward generalization of [28, Theorem 2.6, p. 252].

Proposition 4.3. Let $(X, Y, Z)$ be given, where the matrices satisfy $X^{\top} X=I$, $Y^{\top} Y=I$, and $Z^{\top} Z=I$. Then the problem

$$
\min _{\mathcal{S}}\|\mathcal{A}-(X, Y, Z) \cdot \mathcal{S}\|
$$

has the solution $\mathcal{S}=\mathcal{F}=\mathcal{A} \cdot(X, Y, Z)$.

We will now show that the mode- 1 slices of the tensors $\mathcal{F}$ and $\mathcal{F}_{\perp}^{x}$ can be further orthogonalized. Let

$$
\mathcal{F}=(U, V, W) \cdot \mathcal{F}_{\#}
$$

be the HOSVD of $\mathcal{F}$. It is shown in [5] that the slices of $\mathcal{F}_{\#}$, in each mode separately, are orthogonal (in the inner product induced by the Frobenius norm). In addition, the slices in each mode separately are ordered from largest to smallest (in Frobenius norm). These are the all-orthogonality conditions of the HOSVD [5]. Thus, we can write the rank- $(p, q, r)$ approximation as

$$
(X, Y, Z) \cdot \mathcal{F}=\left(X_{\#}, Y_{\#}, Z_{\#}\right) \cdot \mathcal{F}_{\#}, \quad\left(X_{\#}, Y_{\#}, Z_{\#}\right)=(X U, Y V, Z W) .
$$

Copyright $@$ by SIAM. Unauthorized reproduction of this article is prohibited. 
Note that this transformation does not move the stationary point on the manifold $\mathrm{Gr}^{3}$. We simply use another representative of the equivalence class. Nor does the transformation change the orthogonality relation in Proposition 4.1, which is independent of the choice of representative for the equivalence classes.

Next consider the symmetric positive semidefinite matrix

$$
\left\langle\mathcal{F}_{\perp}^{x}, \mathcal{F}_{\perp}^{x}\right\rangle_{-1}=\left\langle\mathcal{A} \cdot\left(X_{\perp}, Y, Z\right), \mathcal{A} \cdot\left(X_{\perp}, Y, Z\right)\right\rangle_{-1} .
$$

It can be diagonalized by an orthogonal transformation, and the diagonal elements can be ordered from largest to smallest:

$$
\begin{aligned}
Q^{\top}\left\langle\mathcal{A} \cdot\left(X_{\perp}, Y, Z\right), \mathcal{A} \cdot\left(X_{\perp}, Y, Z\right)\right\rangle_{-1} Q & =\left\langle\mathcal{A} \cdot\left(\widetilde{X}_{\perp}, Y, Z\right), \mathcal{A} \cdot\left(\widetilde{X}_{\perp}, Y, Z\right)\right\rangle_{-1} \\
& =\operatorname{diag}\left(\lambda_{p+1}, \ldots, \lambda_{l}\right)
\end{aligned}
$$

where we have used Lemma 2.1 and set $\widetilde{X}_{\perp}=X_{\perp} Q$. In addition, we assume that the eigenvalues are ordered $\lambda_{p+1} \geq \cdots \geq \lambda_{l} \geq 0$. For notational simplicity we suppress the $x$-dependence in the $\lambda_{i}$. Thus, with this basis for $\mathbb{T}_{X}$, the mode- 1 slices of the tensor $\mathcal{A} \cdot\left(\widetilde{X}_{\perp}, Y, Z\right)$ are orthogonal and ordered from the largest to the smallest. Depending on multilinear ranks, some slices may be equal to zero.

4.2. Second order conditions. We next consider the second order conditions for a stationary point $(X, Y, Z)$ to be a local maximum. Let $(\widetilde{X}, \widetilde{Y}, \widetilde{Z})$ be a nearby point on the manifold, and assume that the geodesic curve between the two points is in the direction of a tangent represented by $\bar{D}=\left(D_{x}, D_{y}, D_{z}\right)$ in local coordinates at $(X, Y, Z)$. The objective for the constrained optimization problem in (3.6) may be written as

$$
\Phi(\widetilde{X}, \widetilde{Y}, \widetilde{Z})=\Phi(X, Y, Z)+\langle\bar{D}, \nabla \widehat{\Phi}\rangle+\frac{1}{2}\langle\bar{D}, \widehat{\mathbf{H}}(\bar{D})\rangle+O\left(\|\bar{D}\|^{3}\right) .
$$

Thus, the Taylor expansion of the objective function in terms of tangents, represented in local coordinates, takes into account the constraints on the arguments and $\widehat{\mathbf{H}}(\cdot)$ may be considered as the Hessian for an unconstrained objective function. Then, it follows [20, pp. 174-176] that if the Hessian is negative definite, i.e.,

$$
\langle\bar{D}, \widehat{\mathbf{H}}(\bar{D})\rangle=\sum_{\nu=x, y, z} \sum_{\mu=x, y, z}\left\langle D_{\nu}, \widehat{\mathbf{H}}_{\nu \mu}\left(D_{\mu}\right)\right\rangle<0,
$$

for any nonzero $\bar{D}=\left(D_{x}, D_{y}, D_{z}\right)$, where $\widehat{\mathbf{H}}(\bar{D})$ is given by (3.21), then a stationary point is a strict local maximum. On the other hand, if a stationary point is a strict local maximum, then the Hessian is negative semidefinite.

From the preceding section we see that it is no restriction to assume that all the mode- 1 slices of the combined tensor

$$
\mathcal{A}_{x}=\left[\begin{array}{c}
\mathcal{F} \\
\mathcal{F}_{\perp}^{x}
\end{array}\right]=\left[\begin{array}{c}
\mathcal{A} \cdot(X, Y, Z) \\
\mathcal{A} \cdot\left(X_{\perp}, Y, Z\right)
\end{array}\right]
$$

are orthogonal. This implies that $\langle\mathcal{F}, \mathcal{F}\rangle_{-1}$ and $\left\langle\mathcal{F}_{\perp}^{x}, \mathcal{F}_{\perp}^{x}\right\rangle_{-1}$ become diagonal matrices, and in turn, the $\widehat{\mathbf{H}}_{x x}$ part of the Hessian (3.22) becomes a diagonal Sylvester operator,

$$
\widehat{\mathbf{H}}_{x x}\left(D_{x}\right)=\left\langle\mathcal{F}_{\perp}^{x}, \mathcal{F}_{\perp}^{x}\right\rangle_{-1} D_{x}-D_{x}\langle\mathcal{F}, \mathcal{F}\rangle_{-1} .
$$

Copyright (C) by SIAM. Unauthorized reproduction of this article is prohibited. 
Denote the diagonal elements

$$
\left[\begin{array}{cc}
\langle\mathcal{F}, \mathcal{F}\rangle_{-1} & 0 \\
0 & \left\langle\mathcal{F}_{\perp}^{x}, \mathcal{F}_{\perp}^{x}\right\rangle_{-1}
\end{array}\right]=\operatorname{diag}\left(\lambda_{1}^{2}, \lambda_{2}^{2}, \ldots, \lambda_{l}^{2}\right),
$$

where, for the moment, we have suppressed the $x$-dependence. The diagonal elements in each group are ordered separately,

$$
\lambda_{1}^{2} \geq \lambda_{2}^{2} \geq \cdots \geq \lambda_{p}^{2} \geq 0, \quad \lambda_{p+1}^{2} \geq \lambda_{p+2}^{2} \geq \cdots \geq \lambda_{l}^{2} \geq 0 .
$$

We want to investigate the negative definiteness condition. Therefore we choose $\bar{D}=$ $\left(D_{x}, D_{y}, D_{z}\right)$, where $D_{x}=\left[\begin{array}{ll}d_{1} & d_{2} \cdots d_{r_{1}}\end{array}\right] \neq 0$, and $D_{y}=0, D_{z}=0$. Then at a strict local maximum we have

$$
\begin{aligned}
\langle\bar{D}, \widehat{\mathbf{H}}(\bar{D})\rangle & =\left\langle D_{x}, \widehat{\mathbf{H}}_{x x}\left(D_{x}\right)\right\rangle=\left\langle D_{x},\left\langle\mathcal{F}_{\perp}^{x}, \mathcal{F}_{\perp}^{x}\right\rangle_{-1} D_{x}-D_{x}\langle\mathcal{F}, \mathcal{F}\rangle_{-1}\right\rangle \\
& =\operatorname{tr}\left(D_{x}^{\top}\left\langle\mathcal{F}_{\perp}^{x}, \mathcal{F}_{\perp}^{x}\right\rangle_{-1} D_{x}-D_{x}^{\top} D_{x}\langle\mathcal{F}, \mathcal{F}\rangle_{-1}\right) \\
& =\sum_{i=1}^{p} d_{i}^{\top}\left\langle\mathcal{F}_{\perp}^{x}, \mathcal{F}_{\perp}^{x}\right\rangle_{-1} d_{i}-\sum_{i=1}^{p} d_{i}^{\top} d_{i} \lambda_{i}^{2} \leq 0 .
\end{aligned}
$$

Choosing the columns of $D_{x}$ as $d_{1}=d_{2}=\cdots=d_{p-1}=0$, and $d_{p}=e_{1}$, we then get

$$
\lambda_{p+1}^{2} \leq \lambda_{p}^{2}
$$

i.e., we have a complete ordering of the slices of the combined tensor (4.9). By analogous derivations for the second and third modes we arrive at the following result.

THEOREM 4.4. Assume that $([X],[Y],[Z])$ is a strict local maximum, with corresponding perpendicular $\left(\left[X_{\perp}\right],\left[Y_{\perp}\right],\left[Z_{\perp}\right]\right)$. Then there exist representatives $(X, Y, Z)$ of the equivalence classes, and representatives for the perpendicular equivalence classes, such that the tensor $\mathcal{A}_{x}$, defined by $\mathcal{A}_{x}(1: p,:,:)=\mathcal{F}$ and $\mathcal{A}_{x}(p+1: l,:::)=\mathcal{F}_{\perp}^{x}$ in (4.9), has ordered and orthogonal mode-1 slices,

$$
\begin{gathered}
\left\|\mathcal{A}_{x}(1,:,:)\right\| \geq \cdots \geq \\
\left\|\mathcal{A}_{x}(p,:::)\right\| \geq\left\|\mathcal{A}_{x}(p+1,:::)\right\| \geq \cdots \geq\left\|\mathcal{A}_{x}(l,:,:)\right\|, \\
\left\langle\mathcal{A}_{x}(i,:,:), \mathcal{A}_{x}(j,:,:)\right\rangle=0, \quad i \neq j .
\end{gathered}
$$

Similarly, the tensors $\mathcal{A}_{y}$ and $\mathcal{A}_{z}$, defined by

$$
\begin{array}{ll}
\mathcal{A}_{y}(:, 1: q,:)=\mathcal{F}, & \mathcal{A}_{y}(:, q+1: m,:)=\mathcal{F}_{\perp}^{y}, \\
\mathcal{A}_{z}(:,:, 1: r)=\mathcal{F}, & \mathcal{A}_{z}(:,:, r+1: n)=\mathcal{F}_{\perp}^{z},
\end{array}
$$

are ordered and orthogonal in the second and third modes, respectively. We can write

$$
\begin{aligned}
\left\|\mathcal{A}_{y}(:, 1,:)\right\| \geq \cdots \geq & \left\|\mathcal{A}_{y}(:, q,:)\right\| \geq\left\|\mathcal{A}_{y}(:, q+1,:)\right\| \geq \cdots \geq\left\|\mathcal{A}_{y}(:, m,:)\right\|, \\
& \left\langle\mathcal{A}_{y}(:, i,:), \mathcal{A}_{y}(:, j,:)\right\rangle=0, \quad i \neq j, \\
\left\|\mathcal{A}_{z}(:,:, 1)\right\| \geq \cdots \geq & \left\|\mathcal{A}_{z}(:,:, r)\right\| \geq\left\|\mathcal{A}_{z}(:,:, r+1)\right\| \geq \cdots \geq\left\|\mathcal{A}_{z}(:,:, n)\right\|, \\
& \left\langle\mathcal{A}_{z}(:,:, i), \mathcal{A}_{z}(:,:, j)\right\rangle=0, \quad i \neq j .
\end{aligned}
$$

Further, if the Hessian is negative definite at the current local maximum $([X],[Y],[Z])$, then we have the following strict inequalities:

$$
\begin{aligned}
\left\|\mathcal{A}_{x}(p,:,:)\right\| & >\left\|\mathcal{A}_{x}(p+1,:,:)\right\|, \\
\left\|\mathcal{A}_{y}(:, q,:)\right\| & >\left\|\mathcal{A}_{y}(:, q+1,:)\right\|, \\
\left\|\mathcal{A}_{z}(:,:, r)\right\| & >\left\|\mathcal{A}_{z}(:,:, r+1)\right\| .
\end{aligned}
$$

Copyright (c) by SIAM. Unauthorized reproduction of this article is prohibited. 
By examining the derivations leading to (4.11), we see that if $\lambda_{p}^{2}=\lambda_{p+1}^{2}$, then the local maximum is not unique. The nonuniqueness is analogous to that in the matrix case, except that here it may affect one single mode only.

COROLlary 4.5. If at a local maximum, any mode-1 slice of $\mathcal{F}$ has the same norm as a mode-1 slice of $\mathcal{F}_{\perp}^{x}$, i.e., $\left\|\mathcal{A}_{x}(i,:,:)\right\|=\left\|\mathcal{A}_{x}(j,:,:)\right\|$ for some $i \leq p$ and $j>p$, then the local maximum is not unique. Corresponding statements hold for the other modes.

Note, however, that unlike the matrix case nonuniqueness does not occur if two slices of $\mathcal{F}$ have the same norm, because our problem is formulated in terms of subspaces, not vectors.

We remark that the complete ordering presented in Theorem 4.4 is valid for a specific representation of the equivalence class $([X],[Y],[Z])$ and a specific representation of the corresponding perpendicular $\left(\left[X_{\perp}\right],\left[Y_{\perp}\right],\left[Z_{\perp}\right]\right)$. But regardless of representation, at a strict local maximum point, every mode- 1 slice of $\mathcal{F}$ has a larger norm than any mode- 1 slice of $\mathcal{F}_{\perp}^{x}$. In terms of $\mathcal{A}_{x}$ we have that $\|\mathcal{A}(i,:,:)\|>\|\mathcal{A}(j,:,:)\|$, where $1 \leq i \leq p$ and $p+1 \leq j \leq l$, and similarly for the other two modes.

Example 4.6. Consider again the matrix case of Example 3.3. We see that the point

$$
X=Y=\left[\begin{array}{llll}
e_{i_{1}} & e_{i_{2}} & \cdots & e_{i_{r}}
\end{array}\right]
$$

is a stationary point, since the conditions (4.4)-(4.6) are satisfied. The requirement for a maximum that the Hessian is negative definite on the tangent space translates into the matrix (3.26),

$$
\left[\begin{array}{cc}
-\Sigma_{1}^{2} \otimes I & \Sigma_{2} \otimes \Sigma_{1} \\
\Sigma_{2} \otimes \Sigma_{1} & -\Sigma_{1}^{2} \otimes I
\end{array}\right]
$$

being negative definite. By a symmetric permutation of the rows and columns we get a block diagonal matrix with $2 \times 2$ blocks

$$
\left[\begin{array}{cc}
-\sigma_{i_{\mu}}^{2} & \sigma_{i_{\mu}} \sigma_{i_{\nu}} \\
\sigma_{i_{\mu}} \sigma_{i_{\nu}} & -\sigma_{i_{\mu}}^{2}
\end{array}\right], \quad 1 \leq \mu \leq r, \quad r+1 \leq \nu \leq n
$$

Thus the matrix (4.13) is negative definite if and only if

$$
\sigma_{i_{\mu}}>\sigma_{i_{\nu}}, \quad 1 \leq \mu \leq r, \quad r+1 \leq \nu \leq n,
$$

i.e., all diagonal elements in $\Sigma_{1}$ must be larger than those in $\Sigma_{2}$.

Looking back at Examples 3.3 and 4.6, we see that in the matrix case the second order conditions for a maximum imply that, loosely speaking, the largest element of $F_{\perp}^{x y}$ is smaller than the smallest nonzero element of $F$. Now, for the tensor case we must ask whether the second order conditions imply that the elements in the $\mathcal{F}_{\perp}^{x y}$, $\mathcal{F}_{\perp}^{x z}$, and $\mathcal{F}_{\perp}^{y z}$ blocks are smaller than elements of $\mathcal{F}$ at a local maximum? The answer is $n o$, as will be seen in the following example, where we construct a tensor whose largest elements in the $\mathcal{F}_{\perp}^{x y}$ block are almost as large as the largest element in $\mathcal{F}$.

Example 4.7. Let $\mathcal{A} \in \mathbb{R}^{3 \times 3 \times 2}$ be the tensor given by

$$
\mathcal{A}(:,:, 1)=\left[\begin{array}{lll}
1 & 0 & 0 \\
0 & \epsilon & 0 \\
0 & 0 & f
\end{array}\right], \quad \mathcal{A}(:,:, 2)=\left[\begin{array}{ccc}
0 & 0 & 0 \\
0 & -\epsilon & 0 \\
0 & 0 & f
\end{array}\right],
$$

Copyright $@$ by SIAM. Unauthorized reproduction of this article is prohibited. 
where $\epsilon$ is small and $f$ will be specified. Consider the rank- $(2,2,2)$ approximation of $\mathcal{A}$ given by

$$
(X, Y, Z)=\left(\left[\begin{array}{l}
I \\
0
\end{array}\right],\left[\begin{array}{l}
I \\
0
\end{array}\right], I\right)
$$

The corresponding $\mathcal{F}=\mathcal{A} \cdot(X, Y, Z)$ block simply becomes

$$
\mathcal{F}(:,:, 1)=\mathcal{A}(1: 2,1: 2,1)=\left[\begin{array}{ll}
1 & 0 \\
0 & \epsilon
\end{array}\right], \quad \mathcal{F}(:,:, 2)=\mathcal{A}(1: 2,1: 2,2)=\left[\begin{array}{cc}
0 & 0 \\
0 & -\epsilon
\end{array}\right],
$$

and we see that $\mathcal{F}_{\perp}^{x}, \mathcal{F}_{\perp}^{y}$, and $\mathcal{F}_{\perp}^{z}$ are all equal to zero. It follows that $(X, Y, Z)$ is a stationary point. The eigenvalues of the matrices $\langle\mathcal{F}, \mathcal{F}\rangle_{-i}, i=1,2,3$, are

$$
\left(\lambda_{1}^{x}, \lambda_{2}^{x}\right)=\left(1,2 \epsilon^{2}\right), \quad\left(\lambda_{1}^{y}, \lambda_{2}^{y}\right)=\left(1,2 \epsilon^{2}\right), \quad \lambda_{1,2}^{z}=\frac{1}{2}\left(1+2 \epsilon^{2} \pm \sqrt{1+4 \epsilon^{4}}\right) .
$$

This implies that each diagonal block of the Hessian is negative definite in itself (i.e., $\left\langle D_{x}, \widehat{\mathbf{H}}_{x x}\left(D_{x}\right)\right\rangle<0$ etc.). Further, it is clear that $\mathcal{F}_{\perp}^{y z}$ and $\mathcal{F}_{\perp}^{x z}$ are zero except

$$
\mathcal{F}_{\perp}^{x y}=\mathcal{A}(3,3,1: 2)=[f f] .
$$

Thus, the only off-diagonal nonzero block that occurs in the Hessian is $\widehat{\mathbf{H}}_{x y}$, and therefore the requirement

$$
\langle\bar{D}, \widehat{\mathbf{H}}(\bar{D})\rangle=\left\langle D_{x}, \widehat{\mathbf{H}}_{x x}\left(D_{x}\right)\right\rangle+\left\langle D_{y}, \widehat{\mathbf{H}}_{y y}\left(D_{y}\right)\right\rangle+2\left\langle D_{x}, \widehat{\mathbf{H}}_{x y}\left(D_{y}\right)\right\rangle<0
$$

determines how large $|f|$ can be at a local maximum. Let $D_{x}=\left[\begin{array}{ll}d_{1}^{x} & d_{2}^{x}\end{array}\right]$ and $D_{y}=$

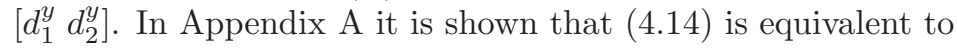

$$
\left[\begin{array}{llll}
d_{1}^{x} & d_{1}^{y} & d_{2}^{x} & d_{2}^{y}
\end{array}\right]\left[\begin{array}{cccc}
-1 & f & 0 & 0 \\
f & -1 & 0 & 0 \\
0 & 0 & -2 \epsilon^{2} & 0 \\
0 & 0 & 0 & -2 \epsilon^{2}
\end{array}\right]\left[\begin{array}{l}
d_{1}^{x} \\
d_{1}^{y} \\
d_{2}^{x} \\
d_{2}^{y}
\end{array}\right]<0
$$

which can easily be seen to be true for $|f|=1-\delta, 0<\delta \leq 1$.

This example shows that at a local maximum there may be elements in the blocks $\mathcal{F}_{\perp}^{x y}, \mathcal{F}_{\perp}^{x z}$, and $\mathcal{F}_{\perp}^{y z}$ that are much larger than the smallest eigenvalues of the matrices $\langle\mathcal{F}, \mathcal{F}\rangle_{-i}, i=1,2,3$. However, the stationary point is not a global maximum if $f$ is close to 1 . By letting the $f$ 's and the $\epsilon$ 's change places by a permutation in the first and second modes, we can make the objective function larger.

5. Perturbation theory. In [4] a first order perturbation analysis of the best approximation problem is performed. Here we will derive perturbation results based on the formulation of the maximization problem as optimization on the product of Grassmann manifolds. In particular, we will generalize the concept of "gap," which determines the sensitivity of the subspaces for the low rank matrix approximation problem, to corresponding tensor quantities that determine the sensitivity of the subspaces in the low multilinear rank approximation problem. First, we will derive the equations governing the sensitivity of a stationary point. Then we consider two special cases to verify that our analysis is sound. Finally we present perturbation bounds for the general case. 
Let $(X, Y, Z)$ be (a representative of) a stationary point on $\mathrm{Gr}^{3}$. After an orthog-

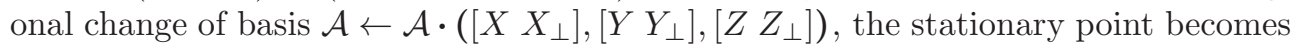

$$
(X, Y, Z):=\left(\left[\begin{array}{l}
I \\
0
\end{array}\right],\left[\begin{array}{l}
I \\
0
\end{array}\right],\left[\begin{array}{l}
I \\
0
\end{array}\right]\right)
$$

where, for simplicity, we have suppressed the dimensions of the identity matrices. In what follows we assume that this change of basis has been performed, and we denote the tensor in this basis by $\mathcal{A}$.

We now make a small perturbation of the tensor, $\widetilde{\mathcal{A}}=\mathcal{A}+\mathcal{E}$, and derive equations for the first order perturbation $(\widetilde{X}, \widetilde{Y}, \widetilde{Z})$ of the stationary point, where $(\widetilde{X}, \widetilde{Y}, \widetilde{Z})$ is another point on the manifold. Then there is a geodesic curve from $(X, Y, Z)$ in some direction $\left(\Delta_{x}, \Delta_{y}, \Delta_{z}\right)$ to $(\widetilde{X}, \widetilde{Y}, \widetilde{Z})$. Consider first the $x$-coordinate, and let $\Delta_{x} \in \mathbb{T}_{X}$ with the thin $\mathrm{SVD}^{5} \Delta_{x}=U \Sigma V^{\mathrm{T}}$. As $U \in \mathbb{T}_{X}$, it can be written in local coordinates, $U=X_{\perp} D_{U}$, where $D_{U}^{\top} D_{U}=I$. The geodesic in the direction $\Delta_{x}$ can now be written [10, section 2.5.1]

$$
X(t)=X V \cos (\Sigma t) V^{\top}+U \sin (\Sigma t) V^{\top}=\left[\begin{array}{c}
V \cos (\Sigma t) V^{\top} \\
0
\end{array}\right]+\left[\begin{array}{c}
0 \\
D_{U} \sin (\Sigma t) V^{\top}
\end{array}\right],
$$

since $\left[X X_{\perp}\right]=I$. By Maclaurin expansion of the trigonometric functions we get

$$
X(t)=\left[\begin{array}{l}
I \\
0
\end{array}\right]+\left[\begin{array}{c}
0 \\
t D_{U} \Sigma V^{\top}
\end{array}\right]+O\left(t^{2}\right) .
$$

Therefore we can write the perturbed stationary point, to first order, as

$$
(\widetilde{X}, \widetilde{Y}, \widetilde{Z})=\left(\left[\begin{array}{c}
I \\
\delta X
\end{array}\right],\left[\begin{array}{c}
I \\
\delta Y
\end{array}\right],\left[\begin{array}{c}
I \\
\delta Z
\end{array}\right]\right)
$$

Parallel transport of the column basis vectors in $X_{\perp}$ in the same direction is given by [10, section 2.5.2]

$$
X_{\perp}(t)=\left(-X V \sin (\Sigma t) U^{\top}+U \cos (\Sigma t) U^{\top}+\left(I-U U^{\top}\right)\right) X_{\perp} \equiv T(t) X_{\perp},
$$

which, after some easy manipulations, becomes

$$
T(t) X_{\perp}=\left[\begin{array}{c}
-t V \Sigma D_{U}^{\top} \\
I
\end{array}\right]+O\left(t^{2}\right) .
$$

It follows that, to first order,

$$
\widetilde{X}_{\perp}=\left[\begin{array}{c}
\delta X_{\perp} \\
I
\end{array}\right]
$$

where $\delta X_{\perp}=-\delta X^{\top}$. Note that $\widetilde{X}^{\top} \widetilde{X}_{\perp}=0$. It is straightforward to derive analogous expressions for $\widetilde{Y}_{\perp}$ and $\widetilde{Z}_{\perp}$.

We now consider the perturbed tensor $\widetilde{\mathcal{A}}$, and require that $(\widetilde{X}, \widetilde{Y}, \widetilde{Z})$ is a stationary point, i.e., the equations corresponding to (4.4)-(4.6) are satisfied, to first order:

$$
\begin{aligned}
& \left\langle\widetilde{\mathcal{A}} \cdot\left(\widetilde{X} \tilde{Y}_{\perp}, \widetilde{Z}\right), \widetilde{\mathcal{A}} \cdot(\widetilde{X}, \widetilde{Y}, \widetilde{Z})\right\rangle_{-1}=0 \\
& \left\langle\widetilde{\mathcal{A}} \cdot\left(\widetilde{X}, \widetilde{Y}_{\perp}, \widetilde{Z}\right), \widetilde{\mathcal{A}} \cdot(\widetilde{X}, \widetilde{Y}, \widetilde{Z})\right\rangle_{-2}=0 \\
& \left\langle\widetilde{\mathcal{A}} \cdot\left(\widetilde{X}, \widetilde{Y}, \widetilde{Z}{ }_{\perp}\right), \widetilde{\mathcal{A}} \cdot(\widetilde{X}, \widetilde{Y}, \widetilde{Z})\right\rangle_{-3}=0 .
\end{aligned}
$$

\footnotetext{
${ }^{5}$ With $\Delta_{x} \in \mathbb{R}^{l \times p}$ the factors of the SVD satisfy $U \in \mathbb{R}^{l \times p}, \Sigma \in \mathbb{R}^{p \times p}$, and $V \in \mathbb{R}^{p \times p}$.
} 
Obviously, since we are performing a first order perturbation analysis of the gradient, we will get a linear system with the Hessian.

THEOREM 5.1. If the stationary point $(X, Y, Z)$ is given by (5.1), and if the point $(\widetilde{X}, \widetilde{Y}, \widetilde{Z})$, given by (5.2), satisfies (5.3) to first order, then

$$
\begin{aligned}
\widehat{\mathbf{H}}_{x x}(\delta X)+\widehat{\mathbf{H}}_{x y}(\delta Y)+\widehat{\mathbf{H}}_{x z}(\delta Z) & =-\left(\left\langle\mathcal{F}_{\perp}^{x}, \mathcal{E}_{0}\right\rangle_{-1}+\left\langle\mathcal{E}_{x}, \mathcal{F}\right\rangle_{-1}\right), \\
\widehat{\mathbf{H}}_{y x}(\delta X)+\widehat{\mathbf{H}}_{y y}(\delta Y)+\widehat{\mathbf{H}}_{y z}(\delta Z) & =-\left(\left\langle\mathcal{F}_{\perp}^{y}, \mathcal{E}_{0}\right\rangle_{-2}+\left\langle\mathcal{E}_{y}, \mathcal{F}\right\rangle_{-2}\right), \\
\widehat{\mathbf{H}}_{z x}(\delta X)+\widehat{\mathbf{H}}_{z y}(\delta Y)+\widehat{\mathbf{H}}_{z z}(\delta Z) & =-\left(\left\langle\mathcal{F}_{\perp}^{z}, \mathcal{E}_{0}\right\rangle_{-3}+\left\langle\mathcal{E}_{z}, \mathcal{F}\right\rangle_{-3}\right),
\end{aligned}
$$

where $\mathcal{F}_{\perp}^{x}, \mathcal{F}_{\perp}^{y}$, and $\mathcal{F}_{\perp}^{z}$ are introduced in (3.19), and

$$
\begin{array}{rlrl}
\mathcal{E}_{0} & =\mathcal{E} \cdot\left(\left[\begin{array}{l}
I \\
0
\end{array}\right],\left[\begin{array}{l}
I \\
0
\end{array}\right],\left[\begin{array}{l}
I \\
0
\end{array}\right]\right), & & \mathcal{E}_{x}=\mathcal{E} \cdot\left(\left[\begin{array}{l}
0 \\
I
\end{array}\right],\left[\begin{array}{l}
I \\
0
\end{array}\right],\left[\begin{array}{l}
I \\
0
\end{array}\right]\right), \\
\mathcal{E}_{y}=\mathcal{E} \cdot\left(\left[\begin{array}{l}
I \\
0
\end{array}\right],\left[\begin{array}{l}
0 \\
I
\end{array}\right],\left[\begin{array}{l}
I \\
0
\end{array}\right]\right), & \mathcal{E}_{z}=\mathcal{E} \cdot\left(\left[\begin{array}{l}
I \\
0
\end{array}\right],\left[\begin{array}{l}
I \\
0
\end{array}\right],\left[\begin{array}{l}
0 \\
I
\end{array}\right]\right) .
\end{array}
$$

The proof is straightforward, but somewhat tedious, so we give it in Appendix B.

Tentatively one might think that the conditioning of the best approximation problem depends on the smallest eigenvalue of the Hessian operator $\widehat{\mathbf{H}}$. However, the presence of the subtensors $\mathcal{F}, \mathcal{F}_{\perp}^{x}, \mathcal{F}_{\perp}^{y}$, and $\mathcal{F}_{\perp}^{z}$ on the right-hand side of (5.4)-(5.6) prevents us from immediately drawing that conclusion. We will first consider two special cases of how to handle this complication, and then analyze the general case.

5.1. Perturbation theory - The matrix case. In this section we will verify that our perturbation analysis is sound by applying it on the low rank matrix approximation problem. In particular, we will show that it gives the perturbation theory for the singular value decomposition. Let the tensor be an $n \times n$ matrix $A$, and consider the maximization problem

$$
\max _{(X, Y) \in \mathrm{Gr}^{2}}\|A \cdot(X, Y)\|=\max _{(X, Y) \in \mathrm{Gr}^{2}}\left\|X^{\top} A Y\right\|,
$$

with $\mathrm{Gr}^{2}=\operatorname{Gr}(n, r) \times \operatorname{Gr}(n, r)$. The solution is given by the truncated singular value decomposition, $(X, Y)=\left(U_{r}, V_{r}\right)$, where the matrices consist of the leading $r$ singular vectors of $A$. This is usually proved using the Courant-Fischer minimax theorem for $A^{\top} A$; see [13, sections 8.1.1 and 8.6.1]. It also follows easily from Examples 3.3 and 4.6 .

Thus, by an obvious change of basis we can consider the perturbation of the diagonal matrix

$$
\left[\begin{array}{cc}
\Sigma_{1} & 0 \\
0 & \Sigma_{2}
\end{array}\right], \quad \Sigma_{1}=\operatorname{diag}\left(\sigma_{1}, \ldots, \sigma_{r}\right), \quad \Sigma_{2}=\operatorname{diag}\left(\sigma_{r+1}, \ldots, \sigma_{n}\right) .
$$

We assume here that $\sigma_{r}>\sigma_{r+1}$. Due to the fact that $\mathcal{F}_{\perp}^{x}$ and $\mathcal{F}_{\perp}^{y}$ are equal to zero, the Hessian perturbation equations (5.4)-(5.6) simplify to

$$
\begin{aligned}
\widehat{\mathbf{H}}_{x x}(\delta X)+\widehat{\mathbf{H}}_{x y}(\delta Y) & =-\left\langle E_{x}, \Sigma_{1}\right\rangle_{-1}, \\
\widehat{\mathbf{H}}_{y x}(\delta X)+\widehat{\mathbf{H}}_{y y}(\delta Y) & =-\left\langle E_{y}, \Sigma_{1}\right\rangle_{-2},
\end{aligned}
$$

which, after reshaping the Hessian operators as in (3.26), can be written as

$$
\left[\begin{array}{cc}
-\left(\Sigma_{1}^{2} \otimes I\right) & \Sigma_{1} \otimes \Sigma_{2} \\
\Sigma_{1} \otimes \Sigma_{2} & -\left(\Sigma_{1}^{2} \otimes I\right)
\end{array}\right]\left[\begin{array}{c}
\operatorname{vec}(\delta X) \\
\operatorname{vec}(\delta Y)
\end{array}\right]=\left[\begin{array}{c}
\left(\Sigma_{1} \otimes I\right) \operatorname{vec}\left(E_{x}\right) \\
\left(\Sigma_{1} \otimes I\right) \operatorname{vec}\left(E_{y}\right)
\end{array}\right]
$$

Copyright $@$ by SIAM. Unauthorized reproduction of this article is prohibited. 
Note, however, that here the matrices $\Sigma_{1}$ and $\Sigma_{2}$ consist of ordered elements. This linear system can be uncoupled (as in Example 4.6) into many $2 \times 2$ systems, of which the following is the "worst" from the point of view of sensitivity:

$$
\left[\begin{array}{cc}
-\sigma_{r}^{2} & \sigma_{r} \sigma_{r+1} \\
\sigma_{r} \sigma_{r+1} & -\sigma_{r}^{2}
\end{array}\right]\left[\begin{array}{l}
\delta x \\
\delta y
\end{array}\right]=-\left[\begin{array}{c}
\sigma_{r} e_{x} \\
\sigma_{r} e_{y}
\end{array}\right] .
$$

To clarify the presentation, we have simplified the notation. The solution is

$$
\left[\begin{array}{l}
\delta x \\
\delta y
\end{array}\right]=\frac{1}{\left(\sigma_{r}-\sigma_{r+1}\right)\left(\sigma_{r}+\sigma_{r+1}\right)}\left[\begin{array}{l}
\sigma_{r} e_{x}+\sigma_{r+1} e_{y} \\
\sigma_{r+1} e_{x}+\sigma_{r} e_{y}
\end{array}\right],
$$

which, using $\left|\sigma_{r} e_{x}+\sigma_{r+1} e_{y}\right| /\left(\sigma_{r}+\sigma_{r+1}\right) \leq\left(\left|e_{x}\right|+\left|e_{y}\right|\right)$, and an analogous inequality for the second row, gives the estimates

$$
\begin{aligned}
& |\delta x| \leq \frac{1}{\sigma_{r}-\sigma_{r+1}}\left(\left|e_{x}\right|+\left|e_{y}\right|\right), \\
& |\delta y| \leq \frac{1}{\sigma_{r}-\sigma_{r+1}}\left(\left|e_{x}\right|+\left|e_{y}\right|\right) .
\end{aligned}
$$

The quantity $\sigma_{r}-\sigma_{r+1}$ is called the gap, and it determines the sensitivity of the singular subspaces. Thus the perturbation theory of the singular value decomposition (see, e.g., [28, Chapter 3]) is a special case of our analysis. Furthermore, it is the difference between the smallest singular value of $\mathcal{F}=A \cdot\left(U_{r}, V_{r}\right)=\Sigma_{1}$ and the largest singular value of $\mathcal{F}_{\perp}^{x y}=A \cdot\left(U_{r \perp}, V_{r} \perp\right)=\Sigma_{2}$ that determines the sensitivity.

5.2. Perturbation theory-The rank- $(1,1,1)$ case. The special case when approximating $\mathcal{A} \in \mathbb{R}^{l \times m \times n}$ with a rank-(1,1,1) tensor, i.e., $p=q=r=1$, gives further insight into the nature of the sensitivity. Here the tensor $\mathcal{F}=\mathcal{A} \cdot(x, y, z)=f$ is a scalar and therefore, from the orthogonality relations (4.4)-(4.6), the subtensors $\mathcal{F}_{\perp}^{x}, \mathcal{F}_{\perp}^{y}$, and $\mathcal{F}_{\perp}^{z}$ are equal to zero, which simplifies (5.4)-(5.6). Obviously, since a change of basis is performed,

$$
(x, y, z)=\left(\left[\begin{array}{c}
1 \\
0 \\
\vdots \\
0
\end{array}\right],\left[\begin{array}{c}
1 \\
0 \\
\vdots \\
0
\end{array}\right],\left[\begin{array}{c}
1 \\
0 \\
\vdots \\
0
\end{array}\right]\right)
$$

is a stationary point. It is straightforward to show that the perturbation equations (5.4)-(5.6) become

$$
\left[\begin{array}{ccc}
-f^{2} I & f F_{\perp}^{x y} & f F_{\perp}^{x z} \\
f F_{\perp}^{y x} & -f^{2} I & f F_{\perp}^{y z} \\
f F_{\perp}^{z x} & f F_{\perp}^{z y} & -f^{2} I
\end{array}\right]\left[\begin{array}{c}
\delta x \\
\delta y \\
\delta z
\end{array}\right]=\left[\begin{array}{l}
f e_{x} \\
f e_{y} \\
f e_{z}
\end{array}\right]
$$

where $F_{\perp}^{x y}=\mathcal{A} \cdot\left(x_{\perp}, y_{\perp}, z\right), F_{\perp}^{x z}=\mathcal{A} \cdot\left(x_{\perp}, y, z_{\perp}\right)$, etc. are now matrices on three of

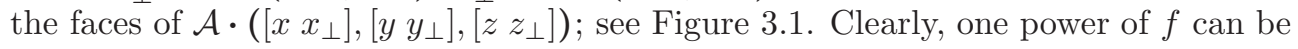
removed from the equation, and we can estimate the norm of the perturbation

$$
\left\|\left[\begin{array}{l}
\delta x \\
\delta y \\
\delta z
\end{array}\right]\right\| \leq\left\|G^{-1}\right\| \cdot\left\|\left[\begin{array}{l}
e_{x} \\
e_{y} \\
e_{z}
\end{array}\right]\right\|,
$$


where

$$
G=-f\left[\begin{array}{ccc}
I & 0 & 0 \\
0 & I & 0 \\
0 & 0 & I
\end{array}\right]+\left[\begin{array}{ccc}
0 & F_{\perp}^{x y} & F_{\perp}^{x z} \\
F_{\perp}^{y x} & 0 & F_{\perp}^{y z} \\
F_{\perp}^{z x} & F_{\perp}^{z y} & 0
\end{array}\right]
$$

If the point $(x, y, z)$ is a strict maximum, then $G$ is negative definite. We have $\left\|G^{-1}\right\|_{2} \leq 1 /\left|\lambda(G)_{\min }\right|$. The eigenvalue of smallest magnitude of $G$ is $\left|\lambda(G)_{\min }\right|=$ $f-\mu_{\max }$, where $\mu_{\max }$ is the largest eigenvalue of

$$
\left[\begin{array}{ccc}
0 & F_{\perp}^{x y} & F_{\perp}^{x z} \\
F_{\perp}^{y x} & 0 & F_{\perp}^{y z} \\
F_{\perp}^{z x} & F_{\perp}^{z y} & 0
\end{array}\right]
$$

In other words, the quantity that determines the sensitivity is

$$
\frac{1}{\text { gap }}=\frac{1}{f-\mu_{\max }} \text {. }
$$

It is worth noting that $\mu_{\max }$ may be larger than the largest singular values of the off-diagonal blocks separately. For instance, with $F_{\perp}^{x y}=F_{\perp}^{x z}=F_{\perp}^{y z}=\rho I$, the largest eigenvalue $\mu_{\max }$ is equal to $2 \rho$.

We see from Figure 3.1 that the result for the matrix case generalizes in the sense that the magnitude of the elements on the three faces $F_{\perp}^{x y}, F_{\perp}^{x z}$, and $F_{\perp}^{y z}$ compared to the magnitude of $f=\mathcal{A} \cdot(x, y, z)$ determines the sensitivity.

We now consider a particular $2 \times 2 \times 2$ tensor $\mathcal{A}(\alpha)$ given by

$$
\mathcal{A}(:,:, 1)=\left[\begin{array}{cc}
10 & 0 \\
0 & \alpha
\end{array}\right], \quad \mathcal{A}(:,:, 2)=\left[\begin{array}{cc}
0 & \alpha \\
\alpha & 0
\end{array}\right], \quad \alpha \geq 0
$$

and its rank- $(1,1,1)$ approximation. This is a symmetric tensor and therefore we will consider a symmetric rank- $(1,1,1)$ approximation. Further, one can easily verify that $\mathcal{A}$ is all-orthogonal. Clearly, the point $\hat{x}=\hat{y}=\hat{z}=\left[\begin{array}{ll}0 & 1\end{array}\right]^{\top}$ is a stationary point corresponding to the global minimum $\Phi(\hat{x}, \hat{y}, \hat{z})=0$. Similarly, and more interestingly, the point $\bar{x}=\bar{y}=\bar{z}=\left[\begin{array}{ll}1 & 0\end{array}\right]^{\top}$ is a stationary point with objective function equal to $\Phi(\bar{x}, \bar{y}, \bar{z})=50$. The matrix governing the sensitivity to perturbations becomes

$$
G=\left[\begin{array}{ccc}
-10 & \alpha & \alpha \\
\alpha & -10 & \alpha \\
\alpha & \alpha & -10
\end{array}\right]
$$

The eigenvalue of smallest magnitude of $G$ is $10-2 \alpha$, which is the gap. One can easily verify that the Hessian $H$ is negative definite for $\alpha<5$, it becomes singular at $\alpha=5$, and for $\alpha>5$ it is indefinite.

Further insight into the properties of the maximization problem is obtained by explicitly considering the objective function $\Phi(x, y, z)=\frac{1}{2}\|\mathcal{A} \cdot(x, y, z)\|^{2}$. Since $\mathcal{A}$ is symmetric, we may set $x=y=z=\left[t \sqrt{1-t^{2}}\right]^{\top}$ for $0 \leq t \leq 1$. Then, maximizing on the product of Grassmann manifolds is equivalent to maximizing the objective function

$$
\Phi(t)=\frac{1}{2}\left(t^{3}(10-3 \alpha)+3 t \alpha\right)^{2}, \quad 0 \leq t \leq 1,
$$


with respect to $t$. In the range $0 \leq \alpha<5$, it is easy to see that $\Phi^{\prime}(t)>0$, so the maximum is attained for $\bar{t}=1$, which implies that $\Phi(\bar{t})=50$ is constant (as a function of $\alpha$ ). Thus the stationary point $(\bar{x}, \bar{y}, \bar{z})$ is a maximum (actually global).

After some elementary calculations one finds that for $5<\alpha \leq 10$ the maximum of the objective function is $\Phi(\alpha)=2 \alpha^{3} /(3 \alpha-10)$ obtained with $t=(\alpha /(3 \alpha-10))^{1 / 2}$. The objective function is larger than 50 , so this is the global maximum. It is straightforward but somewhat tedious to verify that this point is in fact stationary in the sense of Proposition 4.1.

Now assume that $\alpha=5+\epsilon$ for a small $\epsilon>0$. Then we have two stationary points,

$$
\bar{x}=\bar{y}=\bar{z}=\left[\begin{array}{l}
1 \\
0
\end{array}\right], \quad x=y=z=\left[\begin{array}{c}
t \\
\sqrt{1-t^{2}}
\end{array}\right], \quad t=1-\frac{\epsilon}{5}+O\left(\epsilon^{2}\right) .
$$

Thus, for small $\epsilon$ we have two close stationary points that will coincide as we let $\epsilon$ tend to zero. Thus, in this sense the singularity of the Hessian at $\alpha=5$ corresponds to a double stationary point of the objective function.

Further analysis of the geometry of the best approximation problem close to singularities of the Hessian is beyond the scope of this paper. However, we note that in this example, even if the tensor is symmetric, the properties of the tensor problem are similar to those of the nonsymmetric eigenvalue problem, where two almost linearly dependent eigenvectors may develop into a single eigenvector for a Jordan box.

5.3. Perturbation theory-General case. Deriving the perturbation estimate in the general case with multilinear $\operatorname{rank}-(p, q, r)$ is somewhat more involved. We see from the presentation in sections 5.1 and 5.2 that a key ingredient in the analysis is to "divide by $f$ " in order to estimate the right-hand side with only perturbation terms in it. To perform the corresponding operation in the general case we will define an operator that, when acting on (5.4)-(5.6), will make it possible to remove the $\mathcal{F}$, $\mathcal{F}_{\perp}^{x}, \mathcal{F}_{\perp}^{y}$, and $\mathcal{F}_{\perp}^{z}$ factors on the right-hand side of the equations. We will first derive this operation for (5.4). This will lead to the main perturbation results, Theorem 5.3 and Corollary 5.4. Then we discuss how the gap in the matrix singular value case carries over to the tensor case. Finally, we give an example that illustrates that the sensitivity properties for a problem with a low rank tensor plus noise are those that one would expect.

In section 4.2 we showed that at a local maximum we can choose coordinates in such a way that the operator $\widehat{\mathbf{H}}_{x x}$ can be written as a diagonal Sylvester operator ${ }^{6}$ $(4.10)$

$$
\widehat{\mathbf{H}}_{x x}\left(D_{x}\right)=\left(\Lambda_{\perp}^{x}\right)^{2} D_{x}-D_{x}\left(\Lambda^{x}\right)^{2}
$$

where

$$
\begin{aligned}
\left(\Lambda^{x}\right)^{2} & =\langle\mathcal{F}, \mathcal{F}\rangle_{-1}=\operatorname{diag}\left(\left(\lambda_{1}^{x}\right)^{2}, \ldots,\left(\lambda_{p}^{x}\right)^{2}\right) \\
\left(\Lambda_{\perp}^{x}\right)^{2} & =\left\langle\mathcal{F}_{\perp}^{x}, \mathcal{F}_{\perp}^{x}\right\rangle_{-1}=\operatorname{diag}\left(\left(\lambda_{p+1}^{x}\right)^{2}, \ldots,\left(\lambda_{l}^{x}\right)^{2}\right),
\end{aligned}
$$

with ordered diagonal elements and $\left(\lambda_{p+1}^{x}\right)^{2}<\left(\lambda_{p}^{x}\right)^{2}$. We will split $\widehat{\mathbf{H}}_{x x}$ into

$$
\widehat{\mathbf{H}}_{x x}\left(D_{x}\right)=-\mathbf{L}_{x}\left(\mathbf{K}_{x}\left(D_{x}\right)\right)
$$

\footnotetext{
${ }^{6}$ In the terminology of [24] the operator is $\{1,3\}\{2,4\}$-diagonal; cf. (5.15).
} 
where $\mathbf{L}_{x}$ and $\mathbf{K}_{x}$ are (commuting) diagonal Sylvester operators,

$$
\begin{aligned}
\mathbf{L}_{x}\left(D_{x}\right) & =\Lambda_{\perp}^{x} D_{x}+D_{x} \Lambda^{x}, \\
\mathbf{K}_{x}\left(D_{x}\right) & =-\Lambda_{\perp}^{x} D_{x}+D_{x} \Lambda^{x} .
\end{aligned}
$$

Since

$$
\left(\mathbf{L}_{x}\left(D_{x}\right)\right)_{i j}=d_{i j}\left(\lambda_{p+i}^{x}+\lambda_{j}^{x}\right),
$$

it is easy to see that the inverse of $\mathbf{L}_{x}$ may be written as

$$
\mathbf{L}_{x}^{-1}: D_{x} \rightarrow\left\langle\overline{\mathcal{L}}, D_{x}\right\rangle_{1,2 ; 1,2}, \quad \overline{\mathcal{L}}_{i j \mu \nu}=\delta_{i \mu} \delta_{j \nu}\left(\lambda_{j}^{x}+\lambda_{p+i}^{x}\right)^{-1},
$$

where $\delta_{i \mu}$ and $\delta_{j \nu}$ are Kronecker deltas with $1 \leq i, \mu \leq(l-p)$ and $1 \leq j, \nu \leq p$. Also, since $\mathbf{L}_{x}^{-1}$ is a diagonal operator, its norm is equal to the largest diagonal element,

$$
\left\|\mathbf{L}_{x}^{-1}\right\|_{2}=\frac{1}{\lambda_{l}^{x}+\lambda_{p}^{x}} \leq \frac{1}{\lambda_{p}^{x}}=\left\|\mathbf{L}_{p}^{-1}\right\|_{2}
$$

where $\mathbf{L}_{p}^{-1}$ is a linear operator given by $\mathbf{L}_{p}^{-1}: D_{x} \rightarrow \frac{1}{\lambda_{p}^{x}} D_{x}$.

We will let the operator $\mathbf{L}_{x}^{-1}$ act on the right-hand side of (5.4). Considering the first term $\left\langle\mathcal{F}_{\perp}^{x}, \mathcal{E}_{0}\right\rangle_{-1}$ it follows, since $\mathbf{L}_{x}^{-1}$ and $\mathbf{L}_{p}^{-1}$ are diagonal operators, that

$$
\left\|\mathbf{L}_{x}^{-1}\left(\left\langle\mathcal{F}_{\perp}^{x}, \mathcal{E}_{0}\right\rangle_{-1}\right)\right\| \leq\left\|\mathbf{L}_{p}^{-1}\left(\left\langle\mathcal{F}_{\perp}^{x}, \mathcal{E}_{0}\right\rangle_{-1}\right)\right\|=\left\|\left\langle\mathcal{F}_{\perp}^{x} / \lambda_{p}^{x}, \mathcal{E}_{0}\right\rangle_{-1}\right\| \leq c_{1}\left\|\mathcal{E}_{0}\right\|,
$$

where $c_{1}=c_{1}(l, m, n)$ depends only on the dimensions of the tensor $\mathcal{A}$. The last inequality is a consequence of the Cauchy inequality and the fact that each mode- 1 slice of $\mathcal{F}_{\perp}^{x} / \lambda_{p}^{x}$ has a norm strictly less than one. An analogous procedure on the second right-hand term from (5.4) yields

$$
\left\|\mathbf{L}_{x}^{-1}\left(\left\langle\mathcal{E}_{x}, \mathcal{F}\right\rangle_{-1}\right)\right\| \leq\left\|\mathbf{L}_{p}^{-1}\left(\left\langle\mathcal{E}_{x}, \mathcal{F}\right\rangle_{-1}\right)\right\|=\left\|\left\langle\mathcal{E}_{0}, \mathcal{F} / \lambda_{p}^{x}\right\rangle_{-1}\right\| \leq c_{1}\left\|\mathcal{E}_{x}\right\|,
$$

where $c_{1}$ can be taken equal to the constant in the estimate for the first term. We can now treat (5.5) and (5.6) similarly, and we have the following lemma.

Lemma 5.2. Define the operator

$$
\mathbf{L}^{-1}:\left(D_{x}, D_{y}, D_{z}\right) \rightarrow\left(\mathbf{L}_{x}^{-1}\left(D_{x}\right), \mathbf{L}_{y}^{-1}\left(D_{y}\right), \mathbf{L}_{z}^{-1}\left(D_{z}\right)\right),
$$

where $\mathbf{L}_{y}^{-1}$ and $\mathbf{L}_{z}^{-1}$ are analogous to (5.9). Let the right-hand sides in (5.4)-(5.6) be denoted $\bar{R}=\left(R_{x}, R_{y}, R_{z}\right)$. Then

$$
\left\|\mathbf{L}^{-1}(\bar{R})\right\| \leq c\left(\left\|\mathcal{E}_{0}\right\|+\left\|\mathcal{E}_{x}\right\|+\left\|\mathcal{E}_{y}\right\|+\left\|\mathcal{E}_{x}\right\|\right) \equiv E,
$$

where $c$ depends only on the dimensions $l, m, n$, and $\mathcal{E}_{0}, \mathcal{E}_{x}, \mathcal{E}_{y}$, and $\mathcal{E}_{z}$ are defined in Theorem 5.1.

Setting $\bar{D}=(\delta X, \delta Y, \delta Z)$ and $\bar{R}=\left(R_{x}, R_{y}, R_{z}\right)$ as before, we can write the perturbation equations (5.4)-(5.6) as $\widehat{\mathbf{H}}(\bar{D})=\bar{R}$, and their inverted form as

$$
\mathbf{L}(\bar{D})=\mathbf{L} \widehat{\mathbf{H}}^{-1} \mathbf{L} \mathbf{L}^{-1}(\bar{R})=\mathbf{L}^{1 / 2}\left(\mathbf{L}^{-1 / 2} \widehat{\mathbf{H}} \mathbf{L}^{-1 / 2}\right)^{-1} \mathbf{L}^{1 / 2}\left(\mathbf{L}^{-1}(\bar{R})\right) .
$$

The reason for this specific form for the inverted perturbation equations will become evident shortly. We now summarize the derivations and have the following result. 
TheOrem 5.3. Assume that $(X, Y, Z)$ of the form (5.1) is a local maximum of the problem (3.6). Let $\widetilde{\mathcal{A}}=\mathcal{A}+\mathcal{E}$ be a perturbed tensor for small $\mathcal{E}$. Further, let $\mathbf{L}$ be the inverse of the operator in (5.10). Then, the perturbation $\bar{D}=(\delta X, \delta Y, \delta Z)$ on the tangent space of the manifold at $(X, Y, Z)$ can be estimated, to first order, as

$$
\|\bar{D}\| \lesssim \frac{\lambda_{\max }(\mathbf{L})}{\lambda_{\min }(\mathbf{L})} \frac{E}{\lambda_{\min }\left(\mathbf{L}^{-1 / 2} \widehat{\mathbf{H}} \mathbf{L}^{-1 / 2}\right)},
$$

where $\lambda_{\max }(\mathbf{L})=\max \left(\lambda_{1}^{x}, \lambda_{1}^{y}, \lambda_{1}^{z}\right), \lambda_{\min }(\mathbf{L})=\min \left(\lambda_{p}^{x}, \lambda_{q}^{y}, \lambda_{r}^{z}\right)$, and the scalar $E$ is defined in (5.11).

Proof. Take norms in (5.12) and use $\|\mathbf{L}(\bar{D})\| \geq \lambda_{\min }(\mathbf{L})$ and Lemma 5.2.

From Theorem 5.3 we see that the "gap" is proportional to the smallest eigenvalue of $\mathbf{L}^{-1 / 2} \widehat{\mathbf{H}} \mathbf{L}^{-1 / 2}$. In addition, we define the quantity

$$
\kappa(\mathbf{L}):=\frac{\lambda_{\max }(\mathbf{L})}{\lambda_{\min }(\mathbf{L})} \leq \frac{\max \left(\lambda_{1}^{x}, \lambda_{1}^{y}, \lambda_{1}^{z}\right)}{\min \left(\lambda_{p}^{x}, \lambda_{q}^{y}, \lambda_{r}^{z}\right)}
$$

as a "conditioning measure" of the core tensor in the low rank approximation of $\mathcal{A}$.

Corollary 5.4. Let $\mathcal{F}=\mathcal{A} \cdot(X, Y, Z)$ and $\widetilde{\mathcal{F}}=\widetilde{\mathcal{A}} \cdot(\widetilde{X}, \widetilde{Y}, \widetilde{Z})$. Under the same assumptions as in Theorem 5.3,

$$
\widetilde{\mathcal{F}}=\mathcal{F}+\mathcal{E}_{0}+\mathcal{F}_{\perp}^{x} \cdot\left(D_{x}\right)_{1}+\mathcal{F}_{\perp}^{y} \cdot\left(D_{y}\right)_{2}+\mathcal{F}_{\perp}^{z} \cdot\left(D_{z}\right)_{3}+O\left(\|\mathcal{E}\|^{2}\right)=\mathcal{F}+O(\|\mathcal{E}\|) .
$$

Proof. Recalling (5.1) and (5.2) we have

$$
\begin{aligned}
\widetilde{\mathcal{F}} & =(\mathcal{A}+\mathcal{E}) \cdot\left(\left[\begin{array}{c}
I \\
\delta X
\end{array}\right],\left[\begin{array}{c}
I \\
\delta Y
\end{array}\right],\left[\begin{array}{c}
I \\
\delta Z
\end{array}\right]+O\left(\|\mathcal{E}\|^{2}\right)\right) \\
& =(\mathcal{A}+\mathcal{E}) \cdot\left(\left[\begin{array}{l}
I \\
0
\end{array}\right],\left[\begin{array}{l}
I \\
0
\end{array}\right],\left[\begin{array}{l}
I \\
0
\end{array}\right]\right)+\mathcal{A} \cdot\left(\left[\begin{array}{c}
0 \\
\delta X
\end{array}\right],\left[\begin{array}{l}
I \\
0
\end{array}\right],\left[\begin{array}{l}
I \\
0
\end{array}\right]\right) \\
& +\mathcal{A} \cdot\left(\left[\begin{array}{l}
I \\
0
\end{array}\right],\left[\begin{array}{c}
0 \\
\delta Y
\end{array}\right],\left[\begin{array}{l}
I \\
0
\end{array}\right]\right)+\mathcal{A} \cdot\left(\left[\begin{array}{l}
I \\
0
\end{array}\right],\left[\begin{array}{c}
I \\
0
\end{array}\right],\left[\begin{array}{c}
0 \\
\delta Z
\end{array}\right]\right)+O\left(\|\mathcal{E}\|^{2}\right) \\
& =\mathcal{F}+\mathcal{E}_{0}+\mathcal{F}_{\perp}^{x} \cdot\left(D_{x}\right)_{1}+\mathcal{F}_{\perp}^{y} \cdot\left(D_{y}\right)_{2}+\mathcal{F}_{\perp}^{z} \cdot\left(D_{z}\right)_{3}+O\left(\|\mathcal{E}\|^{2}\right),
\end{aligned}
$$

where we have used $\delta X=D_{x}=O(\|\mathcal{E}\|)$ and analogous relations.

We will now verify that the sensitivity of the subspaces depends explicitly on the relation between $\mathcal{F}$ and $\mathcal{F}_{\perp}^{x}, \mathcal{F}_{\perp}^{y}$, and $\mathcal{F}_{\perp}^{z}$, respectively. First we need a few additional definitions. The operator $\mathbf{K}_{x}$, defined in (5.8), has the inverse

$$
\mathbf{K}_{x}^{-1}: D_{x} \rightarrow\left\langle\overline{\mathcal{K}}, D_{x}\right\rangle_{1,2 ; 1,2}, \quad \overline{\mathcal{K}}_{i j \mu \nu}=\delta_{i \mu} \delta_{j \nu}\left(-\lambda_{p+i}^{x}+\lambda_{j}^{x}\right)^{-1},
$$

where $1 \leq i, \mu \leq(l-p)$ and $1 \leq j, \nu \leq p$. Recall that $\|\cdot\|_{2}$ is the operator norm (2.9) associated with the Frobenius "vector" norm. Clearly, since $\mathbf{K}_{x}$ is a diagonal operator,

$$
\left\|\mathbf{K}_{x}^{-1}\right\|_{2}=\frac{1}{-\lambda_{p+1}^{x}+\lambda_{p}^{x}}
$$

Introduce corresponding definitions for $\mathbf{K}$ and $\mathbf{K}^{-1}$ as we did for $\mathbf{L}$ and $\mathbf{L}^{-1}$. Also let $\mathbf{R}$ denote the "off-diagonal blocks" of the Hessian. Using the fact that $\mathbf{K}$ and $\mathbf{L}$ commute, we can make the following reformulation:

$$
\mathbf{L}^{-1 / 2} \widehat{\mathbf{H}} \mathbf{L}^{-1 / 2}=-\mathbf{K}+\mathbf{L}^{-1 / 2} \mathbf{R} \mathbf{L}^{-1 / 2}=-\mathbf{K}^{1 / 2}(\mathbf{I}-\mathbf{S}) \mathbf{K}^{1 / 2},
$$

Copyright $@$ by SIAM. Unauthorized reproduction of this article is prohibited. 
where $\mathbf{S}=\mathbf{K}^{-1 / 2} \mathbf{L}^{-1 / 2} \mathbf{R} \mathbf{L}^{-1 / 2} \mathbf{K}^{-1 / 2}$, and $\mathbf{I}$ is the identity operator. It follows from the negative definiteness of the Hessian at a local maximum that $\mathbf{I}-\mathbf{S}$ is positive definite, which implies that the largest eigenvalue of $\mathbf{S}$ is smaller than 1 . We can then estimate

$$
\left\|\left(\mathbf{L}^{-1 / 2} \widehat{\mathbf{H}} \mathbf{L}^{-1 / 2}\right)^{-1}\right\|_{2} \leq \frac{\left\|\mathbf{K}^{-1}\right\|_{2}}{1-\lambda_{\max }(\mathbf{S})}
$$

Since $\mathbf{K}$ is a diagonal operator, it is clear that

$$
\left\|\mathbf{K}^{-1}\right\|_{2}=\frac{1}{g}, \quad g=\min \left(\lambda_{p}^{x}-\lambda_{p+1}^{x}, \lambda_{q}^{y}-\lambda_{q+1}^{y}, \lambda_{r}^{z}-\lambda_{r+1}^{z}\right),
$$

i.e., $g$ is the minimum gap between $\mathcal{F}$ and $\mathcal{F}_{\perp}^{x}, \mathcal{F}_{\perp}^{y}$, and $\mathcal{F}_{\perp}^{z}$, respectively. The magnitude of $\lambda_{\max }(\mathbf{S})$ depends on the gaps and on the off-diagonal blocks of the Hessian, i.e., on $\mathcal{F}_{\perp}^{x y}, \mathcal{F}_{\perp}^{x z}$, and $\mathcal{F}_{\perp}^{y z}$. From (5.14) we see that a large value of $\kappa(\mathbf{L})$ implies that the gap $g$ is small, but not vice versa.

If the best approximation problem is solved using the Newton-Grassmann method of [12], then, in the computation of the Newton correction, a factorization of the matrix corresponding to the Hessian operator is needed. In addition one can easily solve linear systems involving the operator $\mathbf{L}^{1 / 2}$, using the diagonal form as above. Therefore it is straightforward to compute a numerical value of the quantity $\lambda_{\min }\left(\mathbf{L}^{-1 / 2} \widehat{\mathbf{H}} \mathbf{L}^{-1 / 2}\right)$.

Example 5.5. Assume that the tensor $\mathcal{A}$ is the sum of a low multilinear rank tensor (a "signal tensor") and another tensor, whose elements are of considerably smaller magnitude (a "noise tensor"),

$$
\mathcal{A}=\mathcal{A}_{s}+\mathcal{A}_{n}
$$

In the matrix case, if we know the low rank, the problem of finding the low rank approximation is well conditioned in the sense that the subspaces computed are not sensitive to perturbations of the data, and depend on the gap between the signal and noise singular values; see the discussion in section 5.1.

Assume that we have a low rank approximation of $\mathcal{A}$, and that we have made the transformation described at the beginning of this section, so that the maximum, represented by $(X, Y, Z)$ as in (5.1), is in fact a global maximum. Then, if the low rank approximation has the same dimensions as the multilinear rank of $\mathcal{A}_{s}$, the mass ${ }^{7}$ of the tensor will be concentrated in the subtensor $\mathcal{F}$, while the other parts, $\mathcal{F}_{\perp}^{x}, \mathcal{F}_{\perp}^{y}$, $\mathcal{F}_{\perp}^{z}, \mathcal{F}_{\perp}^{x y}, \mathcal{F}_{\perp}^{x z}$, and $\mathcal{F}_{\perp}^{y z}$, will be small. In this case both $\kappa(\mathbf{L})$ and $\lambda_{\min }\left(\mathbf{L}^{-1 / 2} \hat{\mathbf{H}} \mathbf{L}^{-1 / 2}\right)$ are of moderate magnitude, and the subspaces are insensitive to perturbations of the data: the best low-rank approximation problem is well-conditioned.

On the other hand, if at least one of the ranks is underestimated, then the Hessian is likely to be ill-conditioned, i.e., $\lambda_{\min }\left(\mathbf{L}^{-1 / 2} \widehat{\mathbf{H}} \mathbf{L}^{-1 / 2}\right)$ is large, since one or more of the blocks $\mathcal{F}_{\perp}^{x}, \mathcal{F}_{\perp}^{y}$, and $\mathcal{F}_{\perp}^{z}$ will be large. In this case, $\kappa(\mathbf{L})$ may be small. Similarly, if we have overestimated the actual signal tensor rank, then the core tensor will be ill-conditioned in the sense that $\kappa(\mathbf{L})$ is large. In both of these cases the subspaces are sensitive to perturbations, and the best low rank approximation problem is illconditioned.

\footnotetext{
${ }^{7}$ In the Frobenius norm sense.
} 
6. Conclusion and future work. In this paper we give first and second order conditions for stationarity of the best multilinear rank- $(p, q, r)$ approximation of a tensor, and we develop a first order perturbation analysis. The theory depends heavily on a framework for optimization on a product of Grassmann manifolds. The mathematical soundness of our approach is verified by demonstrating that the perturbation theory for the singular value decomposition is a special case of our analysis. Some similarities and differences between the matrix and the tensor best low rank approximation problems are pointed out.

We anticipate that the perturbation theory will become a vital ingredient in the development of software for the best approximation problem. We also believe that it will be important in the deeper understanding of the mathematical structure of the problem (see the paragraph just before section 5.3). These will be topics in our future research.

Appendix A. Derivation of (4.15).

It is straightforward to show that

$$
\left\langle\widehat{\mathbf{H}}_{x x}\left(D_{x}\right), D_{x}\right\rangle=-\left(d_{1}^{x}\right)^{2}-2 \epsilon^{2}\left(d_{2}^{x}\right)^{2},
$$

and similarly for the other diagonal term. Due to the fact that $\mathcal{F}_{\perp}^{x}$ and $\mathcal{F}_{\perp}^{y}$ are equal to zero the mixed term is

$$
\left\langle\widehat{\mathbf{H}}_{x y}\left(D_{y}\right), D_{x}\right\rangle=\left\langle\left\langle\left\langle\mathcal{F}_{\perp}^{x y}, \mathcal{F}\right\rangle_{3}, D_{y}\right\rangle_{2,4 ; 1,2}, D_{x}\right\rangle .
$$

$\left\langle\mathcal{F}_{\perp}^{x y}, \mathcal{F}\right\rangle_{3}$ is a 4 -tensor, where the first two modes have dimensions equal to one,

$$
\mathcal{C}(1,1,:,:):=\left\langle\mathcal{F}_{\perp}^{x y}, \mathcal{F}\right\rangle_{3}=\left[\begin{array}{ll}
f & 0 \\
0 & 0
\end{array}\right],
$$

and therefore

$$
\left\langle\left\langle\mathcal{F}_{\perp}^{x y}, \mathcal{F}\right\rangle_{3}, D_{y}\right\rangle_{2,4 ; 1,2}=\left[\begin{array}{ll}
f d_{1}^{y} & 0
\end{array}\right] .
$$

We then get

$$
\left\langle\left\langle\left\langle\mathcal{F}_{\perp}^{x y}, \mathcal{F}\right\rangle_{3}, D_{y}\right\rangle_{2,4 ; 1,2}, D_{x}\right\rangle=f d_{1}^{x} d_{1}^{y}
$$

which implies

$$
\begin{aligned}
\langle D, \widehat{\mathbf{H}}(D)\rangle & =\left\langle D_{x}, \widehat{\mathbf{H}}_{x x}\left(D_{x}\right)\right\rangle+\left\langle D, \widehat{\mathbf{H}}_{x x}\left(D_{y}\right)\right\rangle+2\left\langle D_{x}, \widehat{\mathbf{H}}_{x y}\left(D_{y}\right)\right\rangle \\
& =-\left(d_{1}^{x}\right)^{2}-2 \epsilon^{2}\left(d_{2}^{x}\right)^{2}-\left(d_{1}^{y}\right)^{2}-2 \epsilon^{2}\left(d_{2}^{y}\right)^{2}+2 f d_{1}^{x} d_{1}^{y} .
\end{aligned}
$$

This is clearly the same as the expression in (4.15).

Appendix B. Proof of Theorem 5.1.

Proof. Let $\mathcal{A}$ be a given tensor and let $(X, Y, Z)$ be a stationary point for $\Phi(X, Y, Z)$. Introduce the variables

$$
\begin{aligned}
\mathcal{F} & =\mathcal{A} \cdot(X, Y, Z), & & \mathcal{F}_{\perp}^{x}=\mathcal{A} \cdot(X \perp, Y, Z), \\
\mathcal{F}_{\perp}^{y} & =\mathcal{A} \cdot\left(X, Y_{\perp}, Z\right), & & \mathcal{F}_{\perp}^{z}=\mathcal{A} \cdot\left(X, Y, Z_{\perp}\right) .
\end{aligned}
$$

We can assume, without loss of generality, that

$$
X=\left[\begin{array}{l}
I \\
0
\end{array}\right], \quad Y=\left[\begin{array}{l}
I \\
0
\end{array}\right], \quad Z=\left[\begin{array}{l}
I \\
0
\end{array}\right], \quad X_{\perp}=\left[\begin{array}{l}
0 \\
I
\end{array}\right], \quad Y_{\perp}=\left[\begin{array}{l}
0 \\
I
\end{array}\right], \quad Z_{\perp}=\left[\begin{array}{l}
0 \\
I
\end{array}\right],
$$

Copyright (c) by SIAM. Unauthorized reproduction of this article is prohibited. 
where we have suppressed the dimensions of the identity matrices. The perturbed tensor can be written

$$
\widetilde{\mathcal{A}}=\mathcal{A}+\epsilon \mathcal{E}
$$

where $\mathcal{E}$ is a perturbation tensor of norm one and $\epsilon$ is the magnitude of the perturbation. The corresponding stationary point becomes

$$
\widetilde{X}=\left[\begin{array}{l}
I \\
0
\end{array}\right]+\epsilon\left[\begin{array}{l}
\delta X_{1} \\
\delta X_{2}
\end{array}\right], \quad \widetilde{Y}=\left[\begin{array}{l}
I \\
0
\end{array}\right]+\epsilon\left[\begin{array}{l}
\delta Y_{1} \\
\delta Y_{2}
\end{array}\right], \quad \widetilde{Z}=\left[\begin{array}{l}
I \\
0
\end{array}\right]+\epsilon\left[\begin{array}{l}
\delta Z_{1} \\
\delta Z_{2}
\end{array}\right] .
$$

The perturbed stationary point $(\widetilde{X}, \widetilde{Y}, \widetilde{Z})$ is a point in $\mathrm{Gr}^{3}$, and thus it holds that

$$
\tilde{X}^{\top} \tilde{X}=I, \quad \widetilde{Y}^{\top} \tilde{Y}=I, \quad \widetilde{Z}^{\top} \widetilde{Z}=I .
$$

Expanding the first identity $\widetilde{X}^{\top} \widetilde{X}=I$ we obtain

$$
\epsilon\left(\delta X_{1}+\delta X_{1}^{\top}\right)+\epsilon^{2}\left(\delta X_{1}^{\top} \delta X_{1}+\delta X_{2}^{\top} \delta X_{2}^{\top}\right)=0 \quad \Rightarrow \quad \delta X_{1}=O(\epsilon) .
$$

It follows that

$$
\widetilde{X}=\left[\begin{array}{l}
I \\
0
\end{array}\right]+\epsilon\left[\begin{array}{l}
\delta X_{1} \\
\delta X_{2}
\end{array}\right]=\left[\begin{array}{c}
I \\
\epsilon \delta X_{2}
\end{array}\right]+O\left(\epsilon^{2}\right)
$$

Denoting $\delta X=\epsilon \delta X_{2}$, we will write the perturbations (up to first order) by

$$
\widetilde{X}=\left[\begin{array}{c}
I \\
\delta X
\end{array}\right], \quad \widetilde{Y}=\left[\begin{array}{c}
I \\
\delta Y
\end{array}\right], \quad \widetilde{Z}=\left[\begin{array}{c}
I \\
\delta Z
\end{array}\right],
$$

where the two other terms are obtained similarly.

With a similar approach to the perturbed orthogonal complements $\widetilde{X}_{\perp}, \widetilde{Y}_{\perp}, \widetilde{Y}_{\perp}$, we can write (up to first order)

$$
\widetilde{X}_{\perp}=\left[\begin{array}{c}
\delta X_{\perp} \\
I
\end{array}\right], \quad \widetilde{Y}_{\perp}=\left[\begin{array}{c}
\delta Y_{\perp} \\
I
\end{array}\right], \quad \widetilde{Z}_{\perp}=\left[\begin{array}{c}
\delta Z_{\perp} \\
I
\end{array}\right] .
$$

In addition to $\widetilde{X}^{\top} \widetilde{X}=I$ and $\widetilde{X}_{\perp}^{\top} \widetilde{X}_{\perp}=I$ we have the requirement

$$
\widetilde{X}_{\perp}^{\top} \widetilde{X}=\left[\begin{array}{c}
\delta X_{\perp} \\
I
\end{array}\right]^{\top}\left[\begin{array}{c}
I \\
\delta X
\end{array}\right]=\delta X_{\perp}+\delta X=0
$$

yielding $\delta X_{\perp}=-\delta X^{\top}$. Since the perturbed point $(\widetilde{X}, \widetilde{Y}, \widetilde{Z})$ is a stationary point to $\widetilde{A}$, it holds that the corresponding gradient is zero. In particular

$$
\begin{aligned}
& \langle\widetilde{\mathcal{A}} \cdot(\widetilde{X} \perp, \widetilde{Y}, \widetilde{Z}), \widetilde{\mathcal{A}} \cdot(\widetilde{X}, \widetilde{Y}, \widetilde{Z})\rangle_{-1}=0, \\
& \left\langle\widetilde{\mathcal{A}} \cdot\left(\widetilde{X}, \widetilde{Y} \widetilde{Z}_{\perp}\right), \widetilde{\mathcal{A}} \cdot(\widetilde{X}, \widetilde{Y}, \widetilde{Z})\right\rangle_{-2}=0, \\
& \langle\widetilde{\mathcal{A}} \cdot(\widetilde{X}, \widetilde{Y}, \widetilde{Z} \perp), \widetilde{\mathcal{A}} \cdot(\widetilde{X}, \widetilde{Y}, \widetilde{Z})\rangle_{-3}=0 .
\end{aligned}
$$

Copyright (c) by SIAM. Unauthorized reproduction of this article is prohibited. 
We will now analyze (B.1) and remove $O\left(\epsilon^{2}\right)$ terms.

$$
\begin{aligned}
& \left\langle(\mathcal{A}+\epsilon \mathcal{E}) \cdot\left(\left[\begin{array}{c}
\delta X^{\top} \\
I
\end{array}\right],\left[\begin{array}{c}
I \\
\delta Y
\end{array}\right],\left[\begin{array}{c}
I \\
\delta Z
\end{array}\right]\right),(\mathcal{A}+\epsilon \mathcal{E}) \cdot\left(\left[\begin{array}{c}
I \\
\delta X
\end{array}\right],\left[\begin{array}{c}
I \\
\delta Y
\end{array}\right],\left[\begin{array}{c}
I \\
\delta Z
\end{array}\right]\right)\right\rangle_{-1} \\
& =\left\langle\mathcal{A} \cdot\left(\left[\begin{array}{l}
0 \\
I
\end{array}\right],\left[\begin{array}{l}
I \\
0
\end{array}\right],\left[\begin{array}{l}
I \\
0
\end{array}\right]\right), \mathcal{A} \cdot\left(\left[\begin{array}{c}
0 \\
\delta X
\end{array}\right],\left[\begin{array}{l}
I \\
0
\end{array}\right],\left[\begin{array}{l}
I \\
0
\end{array}\right]\right)\right\rangle_{-1} \\
& +\left\langle\mathcal{A} \cdot\left(\left[\begin{array}{l}
0 \\
I
\end{array}\right],\left[\begin{array}{l}
I \\
0
\end{array}\right],\left[\begin{array}{l}
I \\
0
\end{array}\right]\right), \mathcal{A} \cdot\left(\left[\begin{array}{c}
I \\
0
\end{array}\right],\left[\begin{array}{c}
0 \\
\delta Y
\end{array}\right],\left[\begin{array}{l}
I \\
0
\end{array}\right]\right)\right\rangle_{-1} \\
& +6 \text { additional terms }+O\left(\epsilon^{2}\right) \\
& =\left\langle\mathcal{A}_{x}, \mathcal{A}_{x} \cdot(\delta X)_{1}\right\rangle_{-1}+\left\langle\mathcal{A}_{x}, \mathcal{A}_{y} \cdot(\delta Y)_{2}\right\rangle_{-1}+\left\langle\mathcal{A}_{x}, \mathcal{A}_{z} \cdot(\delta Z)_{3}\right\rangle_{-1} \\
& +\left\langle\mathcal{A}_{0} \cdot\left(\delta X_{\perp}\right)_{1}, \mathcal{A}_{0}\right\rangle_{-1}+\left\langle\mathcal{A}_{x y} \cdot(\delta Y)_{2}, \mathcal{A}_{0}\right\rangle_{-1}+\left\langle\mathcal{A}_{x z} \cdot(\delta Z)_{3}, \mathcal{A}_{0}\right\rangle_{-1} \\
& +\left\langle\mathcal{A}_{x}, \mathcal{E}_{0}\right\rangle_{-1}+\left\langle\mathcal{E}_{x}, \mathcal{A}_{0}\right\rangle_{-1}=0
\end{aligned}
$$

where we introduced the terms

$$
\begin{array}{rlrl}
\mathcal{A}_{0} & =\mathcal{A} \cdot\left(\left[\begin{array}{l}
I \\
0
\end{array}\right],\left[\begin{array}{l}
I \\
0
\end{array}\right],\left[\begin{array}{l}
I \\
0
\end{array}\right]\right), & \mathcal{A}_{x}=\mathcal{A} \cdot\left(\left[\begin{array}{l}
0 \\
I
\end{array}\right],\left[\begin{array}{l}
I \\
0
\end{array}\right],\left[\begin{array}{l}
I \\
0
\end{array}\right]\right), \\
\mathcal{A}_{y}=\mathcal{A} \cdot\left(\left[\begin{array}{l}
I \\
0
\end{array}\right],\left[\begin{array}{l}
0 \\
I
\end{array}\right],\left[\begin{array}{l}
I \\
0
\end{array}\right]\right), & \mathcal{A}_{z}=\mathcal{A} \cdot\left(\left[\begin{array}{l}
I \\
0
\end{array}\right],\left[\begin{array}{l}
I \\
0
\end{array}\right],\left[\begin{array}{l}
0 \\
I
\end{array}\right]\right), \\
\mathcal{A}_{x y}=\mathcal{A} \cdot\left(\left[\begin{array}{l}
0 \\
I
\end{array}\right],\left[\begin{array}{l}
0 \\
I
\end{array}\right],\left[\begin{array}{l}
I \\
0
\end{array}\right]\right), & \mathcal{A}_{x z}=\mathcal{A} \cdot\left(\left[\begin{array}{l}
0 \\
I
\end{array}\right],\left[\begin{array}{l}
I \\
0
\end{array}\right],\left[\begin{array}{l}
0 \\
I
\end{array}\right]\right) .
\end{array}
$$

Recalling that $\delta X_{\perp}=-\delta X^{\top}$ and extracting the perturbations from the contracted tensor products using Lemma 2.1, e.g., $\left\langle\mathcal{A}_{x}, \mathcal{A}_{x} \cdot(\delta X)_{1}\right\rangle_{-1}=\left\langle\mathcal{A}_{x}, \mathcal{A}_{x}\right\rangle_{-1} \delta X$, we obtain

$$
\begin{aligned}
& \left\langle\mathcal{A}_{x}, \mathcal{A}_{x}\right\rangle_{-1} \delta X-\delta X\left\langle\mathcal{A}_{0}, \mathcal{A}_{0}\right\rangle_{-1} \\
& +\left\langle\left\langle\mathcal{A}_{x}, \mathcal{A}_{y}\right\rangle_{-1,2}, \delta Y\right\rangle_{4,2 ; 1,2}+\left\langle\left\langle\mathcal{A}_{x y}, \mathcal{A}_{0}\right\rangle_{-1,2}, \delta Y\right\rangle_{2,4 ; 1,2} \\
& +\left\langle\left\langle\mathcal{A}_{x}, \mathcal{A}_{z}\right\rangle_{-1,3}, \delta Z\right\rangle_{4,2 ; 1,2}+\left\langle\left\langle\mathcal{A}_{x z}, \mathcal{A}_{0}\right\rangle_{-1,3}, \delta Z\right\rangle_{2,4 ; 1,2} \\
& =-\left(\left\langle\mathcal{A}_{x}, \mathcal{E}_{0}\right\rangle_{-1}+\left\langle\mathcal{E}_{x}, \mathcal{A}_{0}\right\rangle_{-1}\right)
\end{aligned}
$$

We now see that the left-hand side constitutes exactly the $\widehat{\mathbf{H}}_{x x}(\delta X), \widehat{\mathbf{H}}_{x y}(\delta Y)$, and $\widehat{\mathbf{H}}_{x z}(\delta Z)$ terms from the Hessian; see (3.22) and (3.23). Similar analysis with (B.2) and (B.3) gives the other two equations from Theorem 5.1. The outcome of this theorem is nothing strange and is expected. When perturbing the gradient of an objective function, its Hessian should emerge from the analysis, and that is exactly what happens here.

Acknowledgment. We thank Oleg Burdakov for helpful discussions.

\section{REFERENCES}

[1] B. Bader And T. Kolda, Algorithm 862: MATLAB tensor classes for fast algorithm prototyping, ACM Trans. Math. Software, 32 (2006), pp. 635-653.

[2] P. Comon and B. Mourrain, Decomposition of quantics in sums of powers of linear forms, Signal Processing, 53 (1996), pp. 93-107.

[3] L. De Lathauwer, Signal Processing Based on Multilinear Algebra, Ph.D. thesis, K. U. Leuven, Department of Electrical Engineering (ESAT), Leuven, Belgium, 1997.

Copyright (c) by SIAM. Unauthorized reproduction of this article is prohibited. 
[4] L. De Lathauwer, First-order perturbation analysis of the best rank- $\left(R_{1}, R_{2}, R_{3}\right)$ approximation of a tensor, J. Chemometrics, 18 (2004), pp. 2-11.

[5] L. De Lathaumer, B. De Moor, and J. Vandewalle, A multilinear singular value decomposition, SIAM J. Matrix Anal. Appl., 21 (2000), pp. 1253-1278.

[6] L. De Lathaumer, B. De Moor, and J. Vandewalle, On the best rank-1 and rank$\left(R_{1}, R_{2}, \ldots, R_{N}\right)$ approximation of higher-order tensors, SIAM J. Matrix Anal. Appl., 21 (2000), pp. 1324-1342.

[7] L. De Lathauwer and J. Vandewalle, Dimensionality reduction in higher-order signal processing and rank- $\left(r_{1}, r_{2}, \ldots, r_{n}\right)$ reduction in multilinear algebra, Linear Algebra Appl., 391 (2004), pp. 31-55.

[8] V. DE Silva And L.-H. Lim, Tensor rank and the ill-posedness of the best low-rank approximation problem, SIAM J. Matrix Anal. Appl., 30 (2008), pp. 1084-1127.

[9] G. Eckart And G. Young, The approximation of one matrix by another of lower rank, Psychometrika, 1 (1936), pp. 211-218.

[10] A. Edelman, T. Arias, and S. T. Smith, The geometry of algorithms with orthogonality constraints, SIAM J. Matrix Anal. Appl., 20 (1999), pp. 303-353.

[11] L. EldÉn, Matrix Methods in Data Mining and Pattern Recognition, SIAM, Philadelphia, 2007.

[12] L. EldÉN AND B. SAVAS, A Newton-Grassmann method for computing the best multilinear rank$\left(r_{1}, r_{2}, r_{3}\right)$ approximation of a tensor, SIAM J. Matrix Anal. Appl., 31 (2009), pp. 248-271.

[13] G. H. Golub and C. F. Van Loan, Matrix Computations, 3rd ed., Johns Hopkins Press, Baltimore, MD, 1996.

[14] F. L. HıтCHCOCK, Multiple invariants and generalized rank of a p-way matrix or tensor, J. Math. Phys. Camb., 7 (1927), pp. 39-70.

[15] M. Ishteva, Numerical Methods for the Best Low Multilinear Rank Aapproximation of HighOrder Tensors, Ph.D. thesis, Katholieke Universiteit Leuven, Leuven, Belgium, 2009.

[16] M. Ishteva, L. De Lathauwer, P.-A. Absil, and S. Van Huffel, Differential-geometric Newton method for the best rank- $\left(r_{1}, r_{2}, r_{3}\right)$ approximation of tensors, Numer. Algorithms, 51 (2009), pp. 179-194.

[17] B. N. KhoromskiJ And V. Khoromskaia, Low rank Tucker-type tensor approximation to classical potentials, Cent. Eur. J. Math., 5 (2007), pp. 523-550.

[18] T. G. Kolda And B. W. Bader, Tensor decompositions and applications, SIAM Rev., 51 (2009), pp. 455-500.

[19] L.-H. Lim AND J. MoRTon, Cumulant component analysis: a simultaneous generalization of pca and ica, in Proceedings of Computational Algebraic Statistics, Theories and Applications (CASTA'08), Kyoto University, Kyoto, Japan, 2008.

[20] D. G. Luenberger, Linear and Nonlinear Programming, 2nd ed., Addison-Wesley, Reading, MA, 1984.

[21] J. MorTon, Scalable Implicit Symmetric Tensor Approximation, SIAM Conference on Applied Linear Algebra, Monterey Bay-Seaside, CA, 2009. Preprint presented at the Computational Methods for Tensors minisymposium.

[22] M. Mørup, L. K. Hansen, And S. M. ARnfred, Algorithms for sparse nonnegative tucker decompositions, Neural Computation, 20 (2008), pp. 2112-2131.

[23] L. Omberg, G. H. Golub, And O. Alter, A tensor higher-order singular value decomposition for integrative analysis of DNA microarray data from different studies, Proc. Nat. Acad. Sci., 104 (2007), pp. 18371-18376.

[24] M. RezGhi And L. EldÉn, Diagonalization of tensors with circulant structure, Linear Algebra Appl., 435 (2011), pp. 422-447.

[25] B. SAVAS AND L. EldÉn, Handwritten digit classification using higher order singular value decomposition, Pattern Recognition, 40 (2007), pp. 993-1003.

[26] B. SAVAS AND L.-H. Lim, Quasi-Newton methods on Grassmannians and multilinear approximations of tensors, SIAM J. Sci. Comput., 32 (2010), pp. 3352-3393.

[27] A. Smilde, R. Bro, and P. Geladi, Multi-way Analysis: Applications in the Chemical Sciences, John Wiley \& Sons, New York, 2004.

[28] G. W. Stewart, Matrix Algorithms. Vol II: Eigensystems, SIAM, Philadelphia, 2001.

[29] L. R. TuCKER, The extension of factor analysis to three-dimensional matrices, in Contributions to Mathematical Psychology, Holt, Rinehart, and Winston, New York, 1964, pp. 109-127.

[30] L. R. Tucker, Some mathematical notes on three-mode factor analysis, Psychometrika, 31 (1966), pp. 279-311.

[31] M. A. O. VAsilescu And D. Terzopoulos, Multilinear subspace analysis of image ensembles, in Proceedings of the IEEE Conference on Computer Vision and Pattern Recognition (CVPR'03), Vol. 2, Madison WI, 2003, pp. 93-99.

Copyright (c) by SIAM. Unauthorized reproduction of this article is prohibited. 